\title{
Portrayal and Cartography
}

\section{Paul Hardy \& Kenneth Field, Esri(phardy@esri.com; kfield@esri.com)}

This material is copyright and was published in 2012 as Chapter 11 of the Springer Handbook of Geographic Information, ISBN 978-3-540-72678. The original publication is available at www.springerlink.com. This version should not be publicly hosted, other than at Esri or the authors' own sites.

\section{Introduction}

This chapter summarizes the state of the art in cartography, offers design guidelines as advice on how to maximize the effectiveness of the map as a communications medium, and illustrates how certain characteristics may be better displayed one way over another. Cartography is a huge subject; this chapter does not attempt to be a comprehensive course, nor a definitive text in the subject of cartography (of which there are a number of excellent examples cited in the references section). However, once you have read this chapter, you will find yourself better equipped to understand cartography and be more critically aware of how to represent your information effectively. It also invites you to learn more about cartography as a crucial part of Geographic Information Systems (GIS).

Firstly, the principles and practice of cartography are explored to provide a clear rationale for the importance of map design in communicating the results of geographical analysis. The chapter builds upon earlier detail of geometrical properties of the Earth, its measurement, and transformation from a three-dimensional object to a paper or screen map to show how the choice of map projection is crucial. The construction of maps as a reduced and abstract form of reality allows consideration of some of the constraints on map design and the consequences for how maps are portrayed. Sections on typography and color illustrate the array of possibilities and choices available to the mapmaker and present thoughts on effective map lettering and map coloring. A further section on map organization and layout gives advice on overall map page composition and considers the important components that make up a map. Finally, the design needs of Internet, web, and mobile mapping are considered.

Maps are informative and persuasive tools. Conversely, maps can be notoriously misleading: either intentionally or unintentionally they can lie, or be the outcome of subjective intervention by a cartographer. The information they carry can easily be distorted by cartographic processes, so information from careful analysis may be ineffectively communicated to the intended audience. Maps may be produced for a general purpose audience, or to be used by a very specific user group, and so decisions will need to be taken to ensure the map is fit-for-purpose.

The many choices made in the map-making process, such as feature type (point, line or area), which features to omit for clarity, simplification and generalization of data (e.g. classification of data into class intervals), symbolization (e.g. choice of color scheme for shading thematic maps), or scale of map output can all portray information in vastly different ways and potentially distort the message gained by the user. The principal goal that underlies all map-making is therefore communication - cartography is a language that allows the mapmaker to create graphical components on a map, so that they will be understood by the map reader, resulting in an effective map.

\subsection{What is Cartography?}

Cartography is often defined as the study and practice of making maps. For this chapter, we are concentrating on the practical aspect, which involves a mix of art, craft and science. It has continually evolved to use new technologies through time, of which GIS is just the most recent. Manual techniques that were the mainstay of cartographic design and production as recently as the 1980s have now been replaced by digital workflows.

This had consequences for cartography as new technologies were slow in providing the rich design environments capable of supporting the full range of maps. GIS user interfaces and parameter defaults tended to lead the innocent user to produce garish and cluttered maps, and are only now evolving to take into account the accumulated best practice from cartographic history. As technologies move again, from the Desktop to Server, Mobile and Cloud environments, technological shifts will again have consequences for cartography as it adapts once more. However, the fundamental tenets of design are overarching; technology simply leads to different modes of implementation as well as opening up exciting new avenues for the creation of map-based products. 


\subsubsection{History of Cartography}

Cartography is one of the oldest of human graphical portrayal skills, arguably dating back tens of thousands of years to Stone Age cave drawings showing star maps and ways past landmarks to good hunting locations. From there it progressed through Babylonian clay tablet village plans; Polynesian stick charts for navigation; Egyptian, Greek and Chinese papyrus and parchment maps; medieval Mappa Mundi and portolan navigation charts on vellum; the introduction of paper and printing in the 15th century; to modern computer-printed maps; and increasingly to ephemeral maps displayed on screens of computers and mobile devices. On the way, we have accumulated a wealth of knowledge on best practice of how to make maps of different types and each new advance in technology has brought with it new ways of practicing cartography.

\subsubsection{Relationship between Cartography and GIS}

The word 'cartography' was traditionally used to describe the whole map-making process from survey through compilation to visualization and reproduction. However, in the context of GIS, as a technology predicated on spatial data, cartography as a term is now used more to cover the visualization and reproduction aspects starting from processed data, excluding the earlier stages of building databases and carrying out analysis. As such, GIS is about getting an answer to a geographic question through analyses, while cartography is communicating that answer, particularly to a wider audience than the author. GIS and cartography are complementary, and cartographic tools are now available within GIS to perform visualization and map production tasks.

The relationship between GIS and cartography has not always been so good. In the early days of GIS, with the excitement of the new technology, developers and users forged ahead with computer tools for mapping, without learning from the historical skills of the traditional cartographers, who in turn looked down on the GIS upstarts. The result of this was to create a new breed of geospatial professionals who are more than capable at GIS analysis but who possess little cartographic knowledge or training. This led to poor map design from GIS environments which struggled to provide tools for high quality map work. However, in recent years GIS can be seen as underpinning a re-birth in cartography. The software tools are now capable and powerful and with a greater social appetite for information about the world around us, map design and production has become relevant and important once more. People demand high quality maps and GIS analysts have a responsibility to ensure that they communicate their work effectively using the tools available.

\subsubsection{Good Cartography}

A good map is much more than a visual report of GIS data - a good map has elegance and style, clarity and meaning, as well as appropriate content to the necessary level of precision and detail. The responsibility of the cartographer is to communicate clearly, without clutter or confusion "Perfection is not achieved when nothing can be added, but when nothing more can be taken away" (Antoine de Saint-Exupéry). An extreme example of this is the iconic London Underground map by Harry Beck, which removes all above-ground detail other than the River Thames, dispenses with positional accuracy and scale uniformity in order to show clear connectivity, and through its design, use of color and layout has become a design classic.

There is rarely absolute right or wrong in cartography (though it is true that it is possible to represent data incorrectly). Instead, maps can be viewed as more or less effective. The challenge for the novice cartographer is to learn what constitutes effective cartography so that they can apply the principles in order to make a better map.

\subsection{Types of Maps}

Maps take many different forms and promote a range of cartographic possibilities. Each has specific requirements in terms of their design and construction in order to communicate a message purposefully. These are largely governed by the impact of scale and function. It is actually difficult to categorize maps neatly and many fall into numerous categories but Figure 1 provides a functional distinction for discussion. 


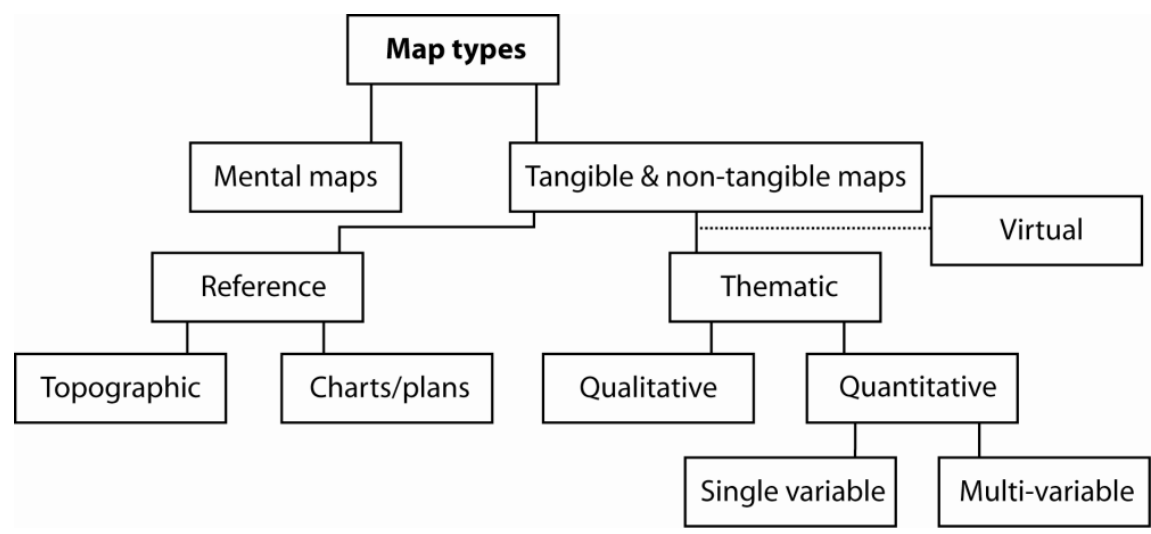

Figure 1: Simplified classification of map types

Mental maps are intangible and reside in our minds. These images are used daily to aid our navigation and recall where places exist in relation to others. The rest of this section concentrates on the more shareable map types arising from use of GIS.

\subsubsection{Topographic Reference Maps}

A reference map (or general-purpose map) displays both natural and man-made objects that exist in the geographical environment. They display the location of objects in space and also in relation to other objects (of the same or different type). Such a map provides a picture of the character of the mapped area and the spatial configuration of objects within it. The topographic map is an example of a reference map designed to illustrate the location of physical features such as coastlines, rivers, forests and lakes along with man-made features such as urban areas and roads. They illustrate the topography of the mapped area (Figure 2).

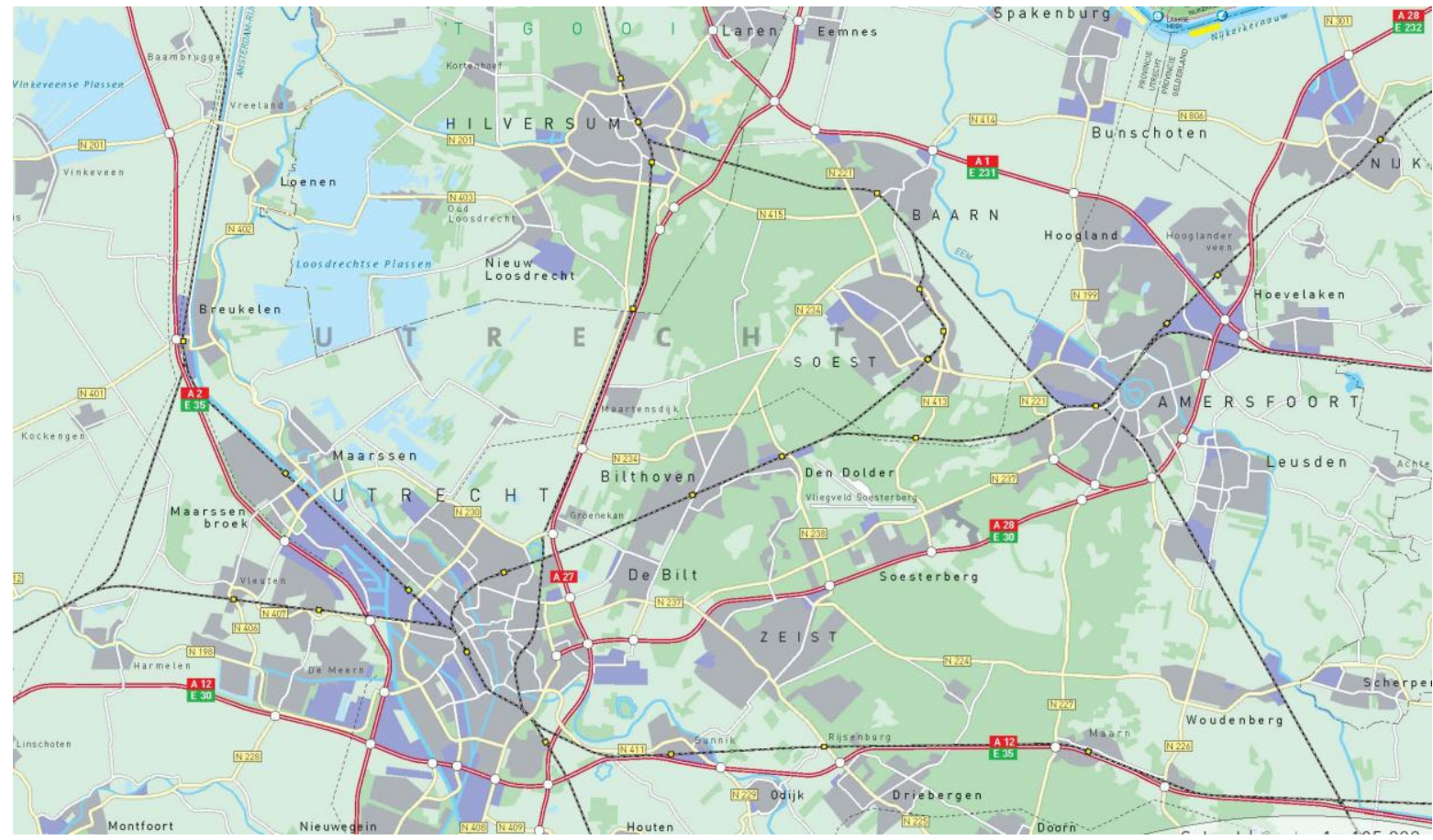

Figure 2. Topographic map extract (Province of Flevoland, courtesy Esri)

Topographic maps also show some features that are non-tangible in the real world yet provide important contexts for describing the character of a region, such as political or administrative boundaries (which may follow the line of a river or a road, but often cuts across country). Small-scale topographic maps would normally be found in atlases and illustrate whole countries or continents. Mid-scale topographic maps would normally form part of a national map sheet series (or dataset) and provide greater detail. 


\subsubsection{Plans}

Maps of a much larger scale are used for special purposes such as site location, where the accuracy, in terms of the positional relationships among the mapped features, is of paramount importance. Such large-scale reference maps are collectively called plans: detailed maps showing buildings, roads and boundary lines. They are often the most detailed available for an area, as they are the result of large-scale survey and data acquisition. Plans are important because the majority of other mapping of the same area, usually at reduced scale, is derived from them. This leads to the term derived mapping. Plans may form the basis of legal documents for determining a property boundary. A plan whose function is based on delimiting property and land ownership zones is termed a cadastral plan.

\subsubsection{Charts}

A chart is a special type of map in that its specific function is to aid navigation. Charts may be used to assist navigation by aircraft (aeronautical charts) or by ships (nautical charts). Topographical features such as the location of mountains or submerged reefs and visible objects such as roads and small islands are often overprinted with additional navigation detail that allows the charts to be worked upon rather than simply viewed. Charts allow users to determine their position and to plot courses.

\subsubsection{Virtual Maps}

Virtual maps are also intangible and usually exist in a computer environment. They can take many forms and many different scales. They are equally effective at illustrating the same range of maps as the tangible form, but require special consideration because the medium of communication differs. The way map users interact with screens differs from their interaction with paper and this also has important implications for the design and production of digital map products.

In particular, maps designed for screen can take advantage of numerous additional effects that are not possible in the printed form. For instance, animation, scrolling, panning and the ability to switch on and off layers are all possible in digital environments. Screen based maps are also increasingly moving away from the planimetric view that many print maps take. Instead of looking down at the surface of the Earth (as a traditional map might), on-screen maps allow tilting of the surface of the Earth to show a pseudo 3-dimensional view with perspective. Such maps tend to be designed to appear as the landscape rather than an abstract version of it and are often based on satellite images or aerial photography draped over a Digital Terrain Model (DTM). Coupled with the ability to navigate around such environments, this sort of 'photorealistic' form of mapping is seeing the creation of innovative, rich web-based applications such as might be seen in 3-D Virtual Philadelphia (Fig 3).

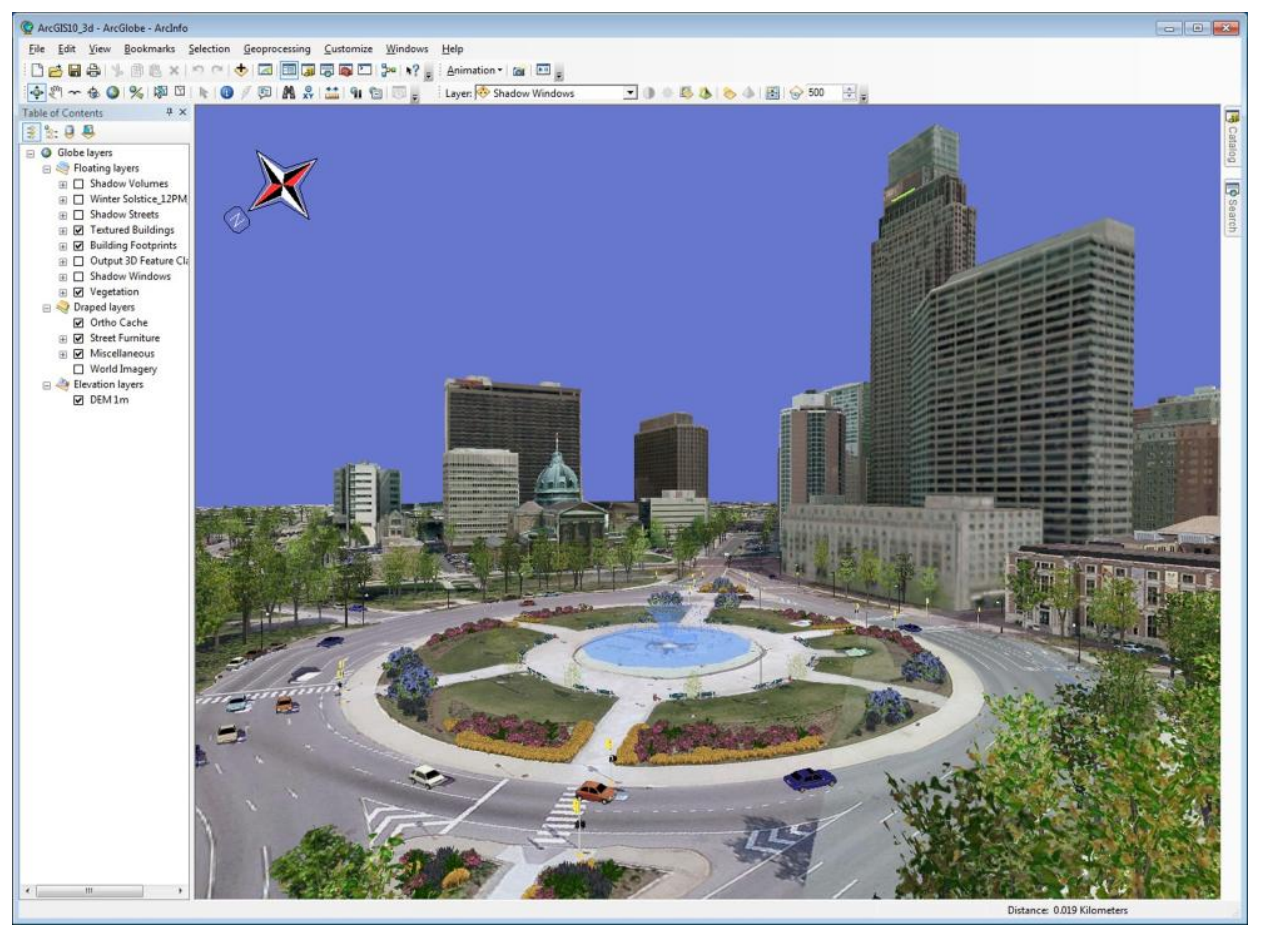

Figure 3. 3D City with virtual buildings (courtesy Esri). 


\subsubsection{Thematic Maps}

Special purpose maps, as opposed to general reference maps, are termed thematic maps. These maps tend to display a single theme of information - hence the term thematic. This may either be by indicating the spatial distribution of a single attribute, or the relationship among many attributes. Thematic maps illustrate the structural characteristics of the geographical distribution of a particular feature or concept. This involves mapping an abstraction of a physical or cultural phenomenon, such as the distance between objects and their directional relationship, patterns of location, or some magnitude of change, perhaps over time. Thematic maps vary considerably but examples include maps depicting precipitation, population distribution, hurricane incidence and pollution. Thematic maps tend to be small scale because the geographical distribution of a theme is often most appropriately shown, and accurately depicted, over a large area. Small scale mapping allows the display of the overall structure of the distribution to become visible.

\subsubsection{Qualitative Thematic Mapping - What is Here?}

Qualitative maps illustrate the spatial distribution of data measured on a nominal scale; that is, data that are similar in kind. For instance, a map showing the location of atmospheric weather stations would be both thematic and qualitative; and would illustrate both location and spatial distribution. At a relatively small scale (e.g. country-wide), this map would be designed to give an indication of the general pattern of distribution of weather stations rather than precise geographical position. It would assist in understanding the locations of measurement of atmospheric conditions to aid interpretation of further thematic maps that display quantitative aspects of the weather.

Figure 4 illustrates a land cover qualitative map with each separate class indicating a separate land use type. There is very little topographic information on this map other than some administrative boundaries to provide structure and spatial location.

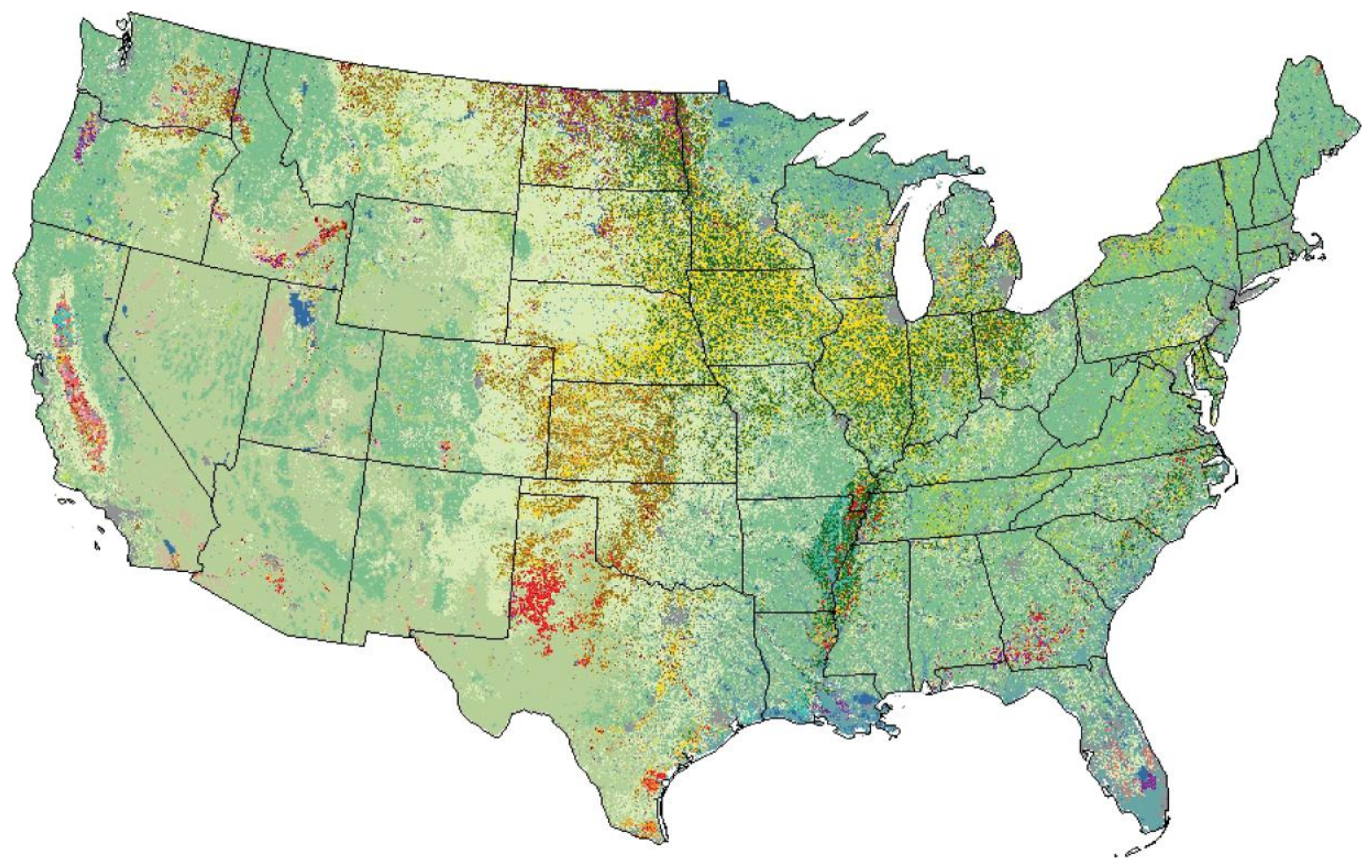

Figure 4. Land cover types (Map Use - courtesy Esri Press)

\subsubsection{Quantitative Thematic Mapping - How Much is Here?}

Quantitative maps display spatial aspects of empirical data. This is normally a single variable displayed thematically though some thematic maps are capable of displaying two (bivariate maps) or more (multivariate maps) variables using particular forms of symbol design. Taking our weather stations, measurements of precipitation may be used to create a map showing the spatial distribution of the amount of rainfall in a 24 hour period. Other thematic maps based on measurements at the weather stations may include temperature and atmospheric pressure. The map displays variation in the theme from place to place, measured on either an ordinal or interval/ratio scale. 
There are many different types of quantitative thematic map, each of which should be used in a particular way and each of which has its own advantages and limitations. Choropleth maps, for instance, are excellent ways of illustrating the spatial distribution of phenomena yet they are only able to show data that is prepared in ratio or proportional format (e.g. persons per sq. mile or percentage of Hispanic population). The purpose of such a map is to allow one area to be visually compared with another so it is important that differences in population (as a denominator) or areal size are accounted for. Figure 5 shows a simple choropleth map identifying the percent of total employment of jobs in foreign owned companies. The map is designed to show that some counties have a higher proportion of the workforce in foreign owned companies than others, illustrated by darker color. The map also shows areas that display similar characteristics.

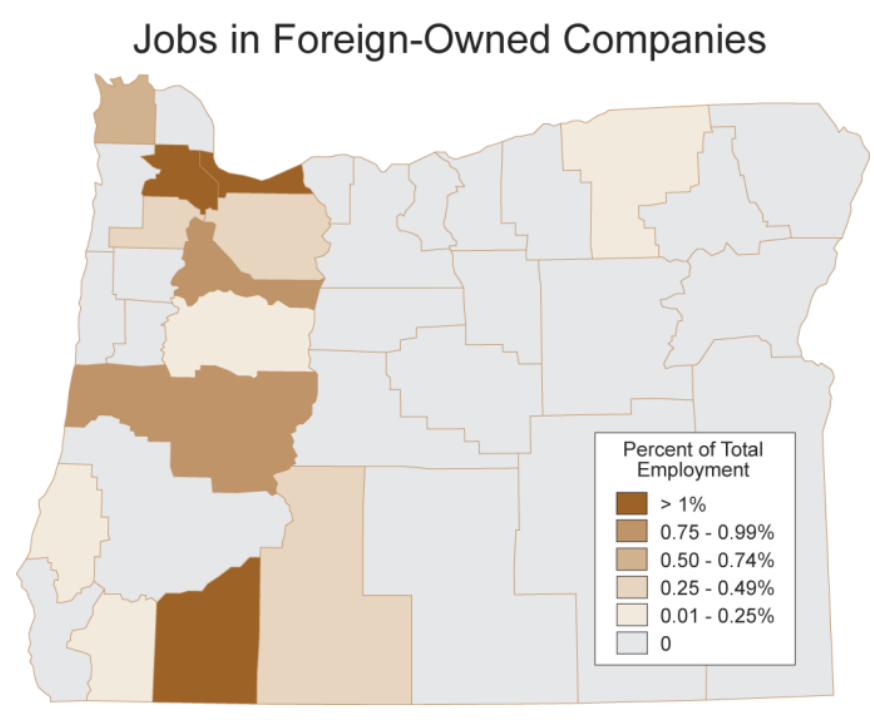

Figure 5. Choropleth map showing percent of jobs in foreign owned companies (Courtesy Esri Press)

An isopleth map is similar to a choropleth except the class intervals are delimited by contours of equal data values rather than being presented using a geographical framework such as the administrative boundaries in Figure 5. Figure 6a illustrates a typical isopleth map where contours have been drawn to show lines of equal value and the categories take on a nested appearance. This sort of map is conventionally used for portraying data that is sampled at points but which represents a continuous surface such as atmospheric pressure or temperature.

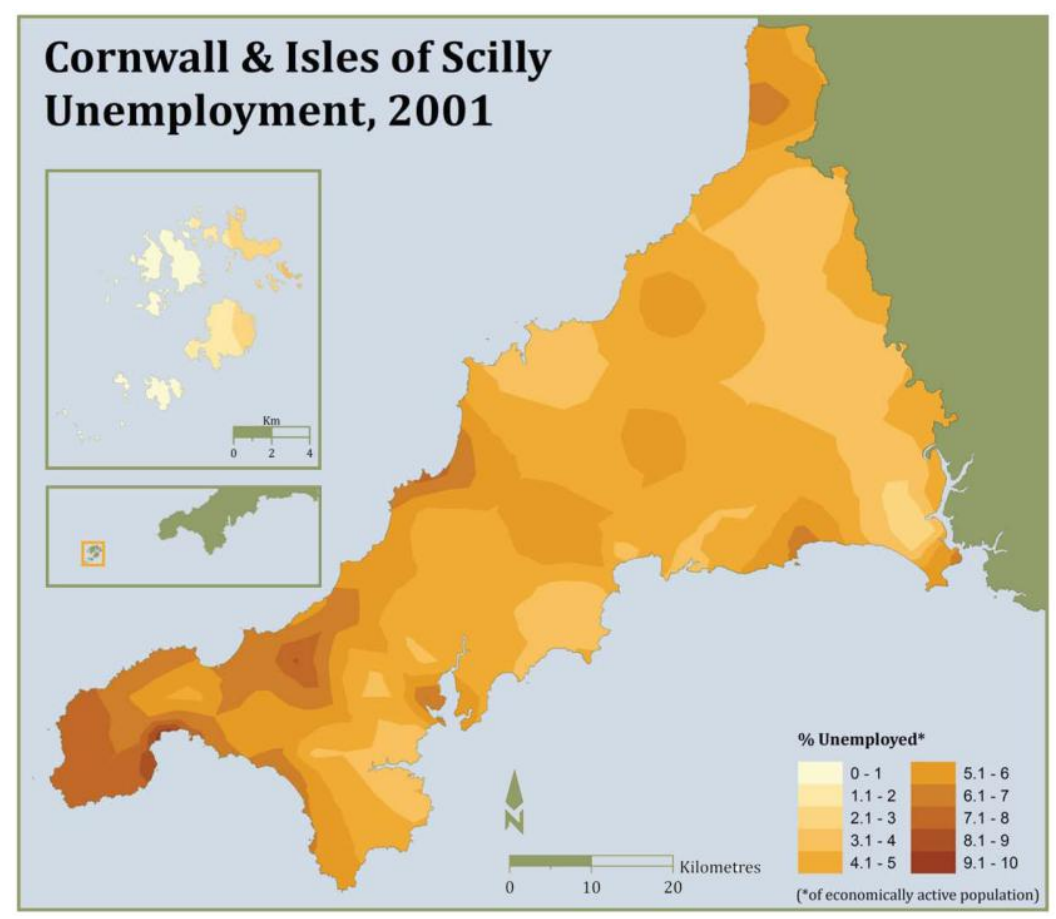

Figure 6a. Isopleth map showing population unemployment (courtesy B. Watson). 
Other types of thematic map are better suited to different data types. For instance, Fig. 6b illustrates absolute data (totals) mapped as proportional symbols and displayed at points within the area concerned and Fig. 7 illustrates linear data mapped as a proportional flow chart.

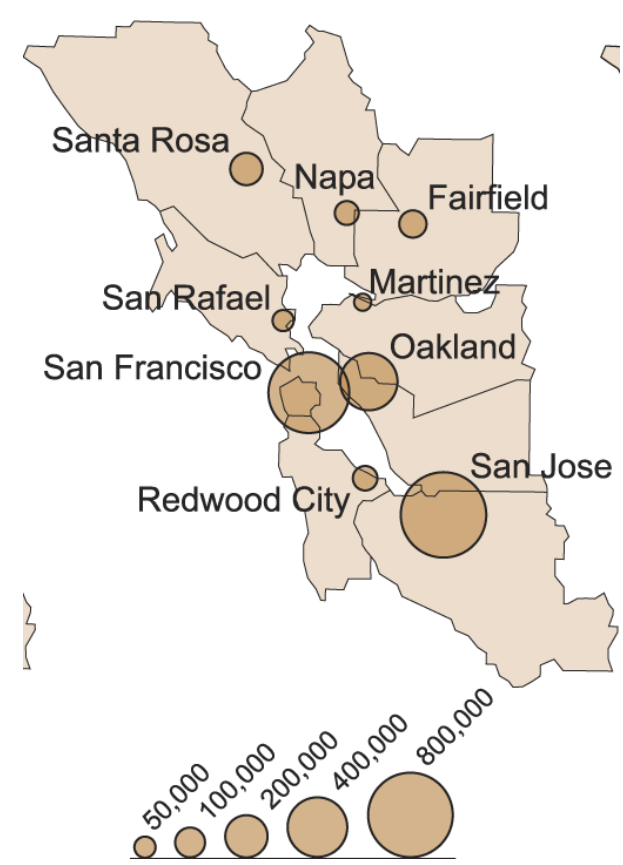

Figure 6b. Proportional Symbol map showing San Francisco Bay Area County Seat Population, 2000 (Courtesy Esri Press)

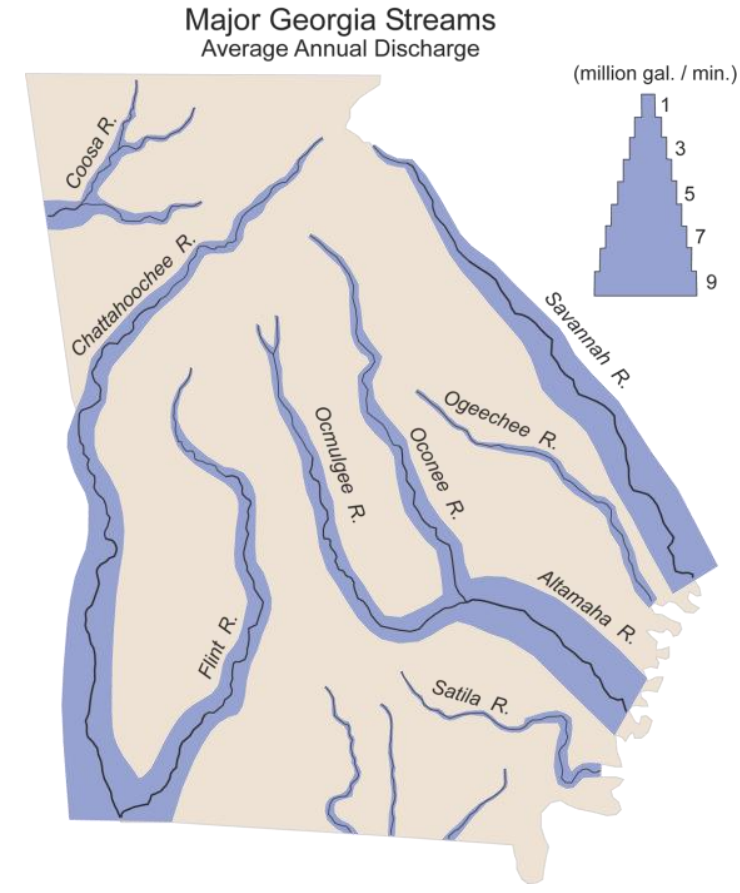

Figure 7. Flow map showing average annual discharge for major Georgia streams (Courtesy Esri Press)

\subsection{Abstraction and Generalization}

All maps are abstractions of the real world. The heart of cartography is to tune that abstraction to suit the particular map, and to the message that it needs to communicate. This means to leave out clutter and to make prominent the things that matter. To achieve this will often mean removing or fading irrelevant detail, while exaggerating foreground information.

\subsubsection{Scale}

Any map has an associated scale. This is a fraction, usually expressed as a ratio, e.g., 1:250,000. What this means is that most features on the map are at $1 / 250,000^{\text {th }}$ of their actual size. Scale is often casually abbreviated to just the denominator, e.g., a $250 \mathrm{~K}$ map, but note that a small-scale map means a smaller fraction, so has a bigger denominator - a large scale map (1:1000) shows a small area but with a large amount of detail, but a small scale map $(1: 250,000)$ shows a large area with a small amount of detail.

\subsubsection{Resolution}

Resolution is a related concept to scale, being the number of resolvable things per unit distance. It applies to raster images used as source (e.g. hill shading images), often measured in pixels per mm, and to output media, such as computer screens measured in dots per inch (dpi). The output resolution has an impact on the design and choice of appropriate symbology, not least because you cannot draw a line narrower than the output resolution. The input resolution should match the output resolution - too coarse an input resolution will appear blocky, while too fine will be sub-sampled on output and may lose critical detail.

Resolution is also a term applied to vector data, describing the smallest resolvable size (e.g. minimum size to show a building as a polygon), or the smallest distance between two lines so that the eye can separate them. When data captured at larger scale is used for small-scale mapping, the data may need to be generalized to meet the resolution constraints. 


\subsubsection{Generalization}

Typically, many features on a map will often have to be exaggerated in size to make them clearly visible. So, for example, a major road on a $250 \mathrm{~K}$ road map will often be symbolized $2 \mathrm{~mm}$ wide for readability, even though strict scale would imply that the corresponding real road is $500 \mathrm{~m}$ wide! To make space for clear feature symbolization may well require that the map space be distorted, and important objects (a lake, a church) close to the road may have to be moved away in order that they can be visible. It is more important to see that there is a lake on the left of the road, than to know its precise position. This process of abstraction is known as generalization - it involves lying about reality, in order to communicate the essential information. The main operations used are:

- Selection - suppressing irrelevant detail, while including the important features, e.g. dropping certain feature classes or applying a database filter selection based on attributes or feature size.

- Simplification - reducing the complexity of features, e.g. by removing excess points from lines.

- Aggregation - combining similar adjacent features, e.g. joining several small woodlands to make up a forested area.

- Exaggeration - Increasing the size of features to meet readability criteria, e.g. minimum area for buildings, or minimum width for road lines.

- Typification - removal of excess features in a location, while keeping some examples to show the type of feature present, e.g., keeping (and enlarging and simplifying) a subset of buildings down a road to show that buildings are present.

- Displacement - moving features away from their true position, to keep them legible, particularly when exaggeration has been applied to them or to neighbors.

Generalization is not an easy task, and has resisted full automation. Most GIS have tools for per-feature geometric simplification (e.g. Douglas-Peucker line simplification). Recent GIS advances have introduced a contextual generalization engine and set of tools, such as those in the 1Spatial Clarity product, or the optimization engine and contextual cartography tools for road and building displacement in Esri ArcGIS 10.

\subsubsection{Map Projections}

To state the obvious: the world is curved, while paper (and a computer screen) is flat. In addition, positional information is increasingly recorded (from GPS etc) into GIS databases as measurements of Latitude and Longitude angles from the center of the Earth. These are usually presented in ellipsoidal degrees in the WGS84 datum (see chapter 9 on Geodesy for details of such spherical coordinates), while the mathematics of GIS calculation and the output of cartography require Cartesian $(\mathrm{X}, \mathrm{Y})$ planar coordinates.

Many GIS users can and do ignore this fact, particularly if their maps cover a small area not near the poles. They either rely on the database data being already transformed and stored in a suitable projection for that part of the world (e.g. US State Plane feet, or British National Grid meters), or they just use the spherical angles as if they were linear distances and hope to get away with the fact that while a degree of longitude stays the same size, a degree of latitude gets smaller as you move away from the equator. In southern Norway or Alaska, at 60 degrees north, the ratio is two to one, and by 84 degrees it is ten to one! Maps that use Lat/Long as if they were $\mathrm{X}, \mathrm{Y}$ are actually using an implicit projection called 'plate carrée', and the distortions of size and shape can be very significant.

However the educated cartographer needs to make better choices, particularly for maps of larger countries, continents and the world. All GIS software provides coordinate transformation tools to convert from Lat/Long to projected $\mathrm{X}, \mathrm{Y}$, either for storage in the database, or dynamically on output of a map visualization.

There are a myriad of projections that have been developed over the centuries, too many to cover in detail here. See web resources such as http://en.wikipedia.org/wiki/List_of_map_projections, or standard textbooks such as [Snyder 1997].

One old and well-known example is the Mercator projection (Fig. 8) - think of it as wrapping a big cylinder of paper around the Earth, so that it touches at the Equator. Put a bright light in the center of the Earth, and project out through the Earth's surface onto the paper. Cut the paper along a line of longitude, and flatten it out. Scale it down linearly until it fits onto your map sheet! 


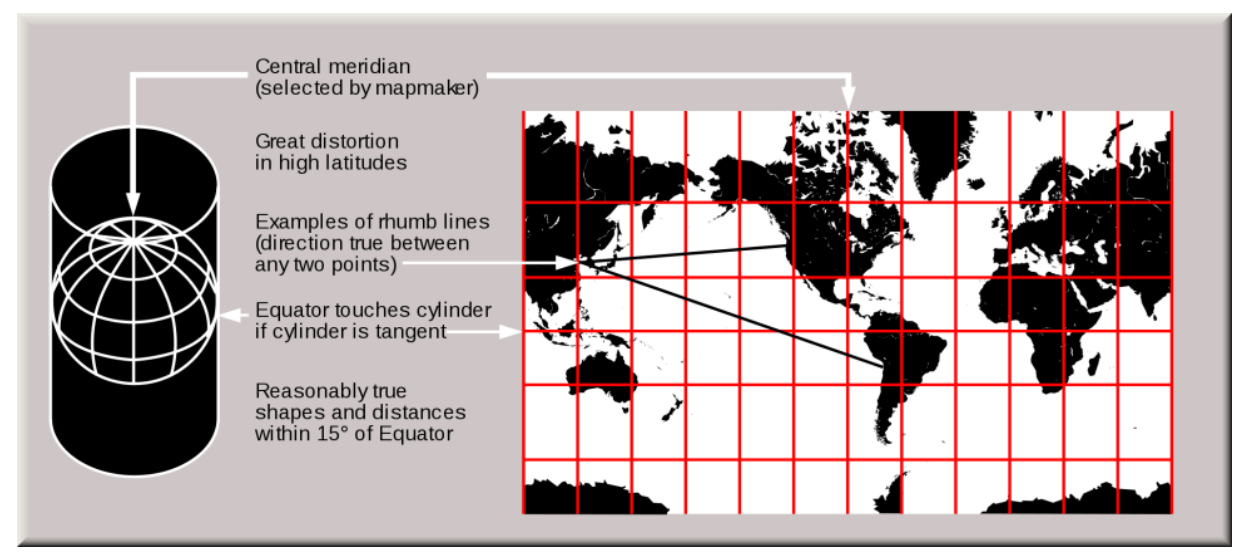

Figure 8. Mercator map projection

Mercator is good for navigation, because lines of both longitude and of latitude are straight lines on the map. It is bad for many other purposes, because it exaggerates the size of features at high latitudes (Australia is really bigger than Greenland), and distorts the shape of large features.

There is no such thing as a perfect projection - any choice has to be a compromise as it will preserve some properties of the round Earth but distort others. Projections are often classified into

- Preserving direction (azimuthal)

- Preserving shape locally (conformal)

- Preserving area (equal-area)

- Preserving distance (equidistant)

- Preserving shortest route (gnomonic)

When designing a map, think "I can't preserve both shape and area - so which is more important for the cartography of this map - conformal or area-preserving?"

One example where insufficient thought about projections produced a misleading map arose in 2003, when a British magazine "The Economist" published a map using Mercator projection, supposedly showing the countries within range of North-Korean missiles, indicating that the USA and Europe were outside the range of both classes of Taepodong missiles (Figure 9a). A few days later, they re-issued the map, still on Mercator, but with the ranges correctly shown, with the USA and Europe well within range (Figure 9b)!

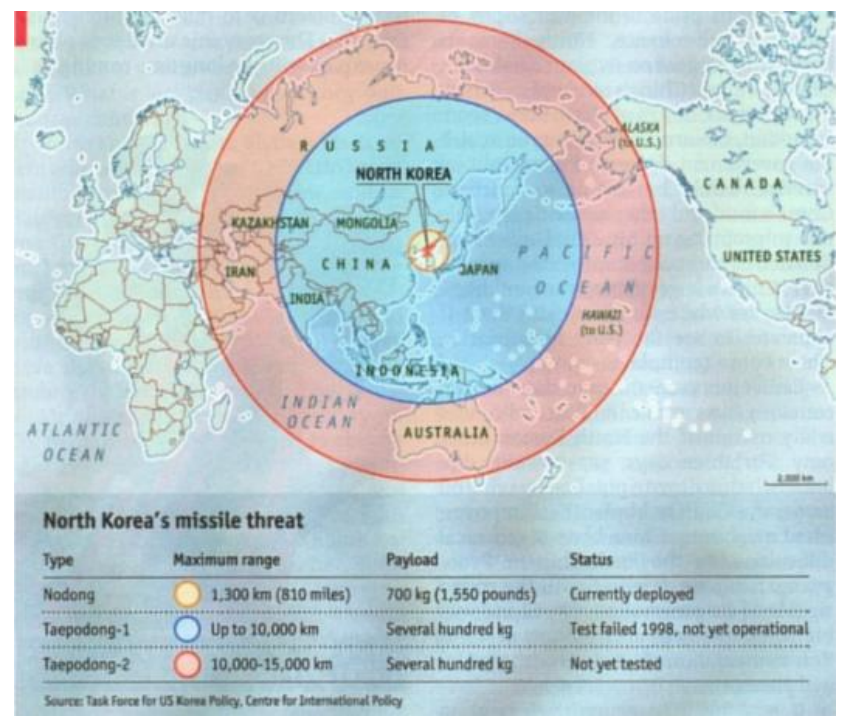

Figure 9a. May $3^{\text {rd }} 2003$ map - The Economist. Original map of North Korean missile range

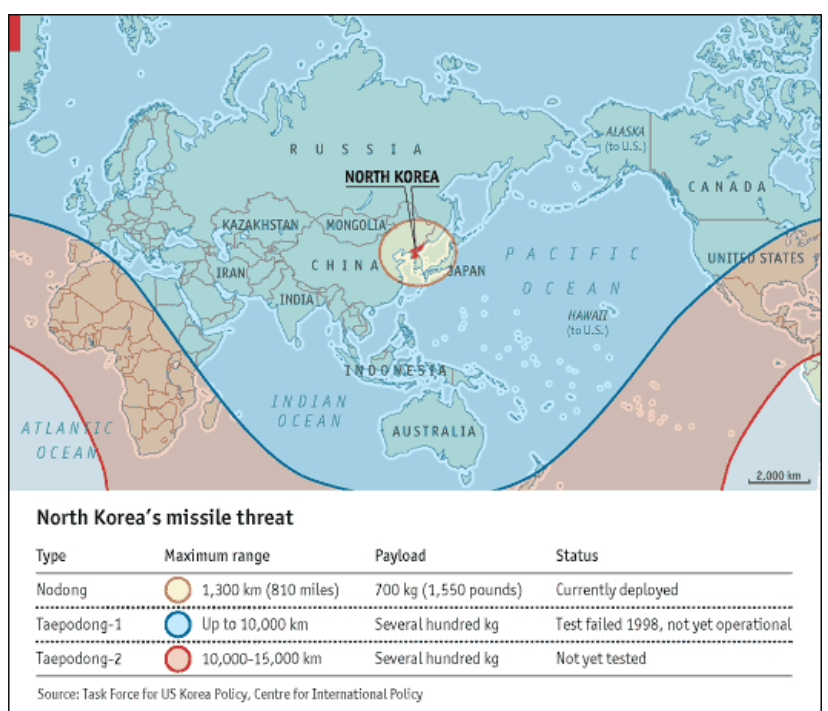

Figure 9b. May $17^{\text {th }} 2003$ map - The Economist. Corrected map of North-Korean missile range

A better alternative might have been to show the world in a stereographic projection centered on North Korea, so that the ranges could be drawn as intuitive circles. An example is shown in Fig. 9. 


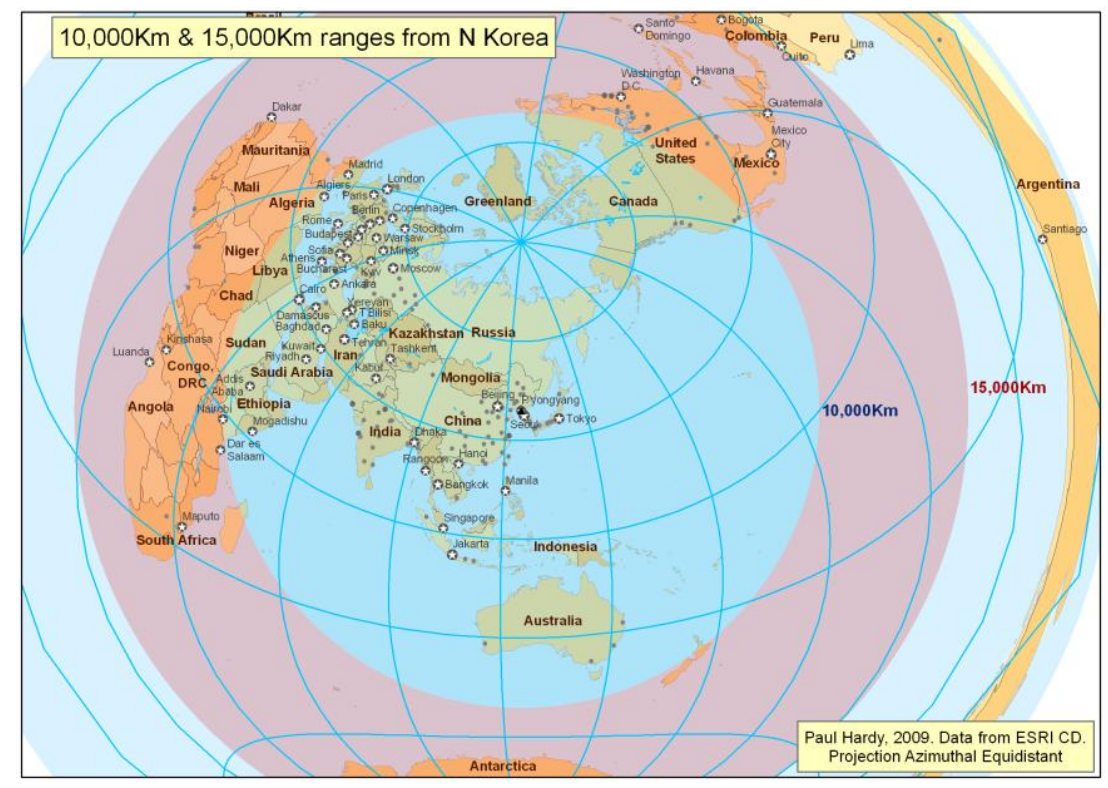

Figure 10. Re-drawn threat of North-Korean missiles on a stereographic projection centered on North Korea

\subsection{Map Symbol Design}

The way in which we represent features and their attributes is through the design and placement of graphical signs. These signs do not necessarily take on the appearance of the object in reality but are used to represent the object. The signs should have meaning to enable the map reader to interpret them accurately and appropriately relate them to the real-world object.

The fundamental operations of generalization take care of many of the processes that apply to depicting features graphically with specific reference to scale as a constraining factor. Symbolization extends these processes by applying graphical treatments for representing features on a map. Symbols are used in a variety of ways to imbue the map with meaning and to convey a message.

\subsubsection{Dimensionality}

Geographical phenomena exist in reality at different levels of dimensionality:

- Point (zero-dimensional)

- Line (one-dimensional)

- Area (two-dimensional)

- $\quad$ Surface $\left(2 \frac{1}{2} 2_{\text {-dimensional })}\right.$

- Volume (three-dimensional)

- Space-time (four-dimensional)

Each dimension of geography requires different handling for symbology. For instance, a phenomenon that exists as a linear feature in reality (e.g. a road) may be equally represented by a linear object in a cartographic sense. However, spatial dimensions do not always map neatly in this way since scale and form are intricately linked. As scale and the level of enquiry alter, the spatial dimension of a feature may also change. For instance, at a meso-scale, a city may be represented as an area with two-dimensionality. However, at a macro-scale the same city might be better represented as a point feature with zero-dimensionality. Map scale therefore plays an extremely important role in determining how the spatial dimension of geographical phenomena are handled and will impact upon how they are represented cartographically.

Multi-dimensional cartography is challenging: many 3-D phenomena have four variables: $x$ and $y$ coordinates, a $z$ coordinate indicating height or depth and the value of the phenomena at that point. Atmospheric pollution may be measured in such a way. For instance, values of nitrogen dioxide can be measured at a point in $x, y$ space and also at a $z$ point above ground level giving a value that is unique and which might differ from values taken at different $z$ heights at the same $x, y$ coordinate. Alternatively, values of nitrogen dioxide may differ temporally for the same $(x, y, z)$ point. This would give rise to a fourth dimension that includes time as a unique characteristic of the phenomenon. 


\subsubsection{Visual Variables}

Once we know how a feature is represented and what measurement is associated with it then we turn our attention to designing a symbol set appropriate for that particular combination. Visual variables are used to provide a palette from which a cartographer can select in order to represent perceived differences on a map. Many of the visual variables discussed here are particularly appropriate for thematic mapping but some are also used in topographic mapping. They were first described by Jacques Bertin [Bertin 1983].

Figure 11 illustrates the range of visual variables. The columns in the figure represent the feature dimension; rows identify individual visual variables. Each cell in the figure illustrates the application of the visual variable for that particular dimension. By varying the graphical treatment of the symbol it is possible to attribute the symbol with meaning that reflects the data being mapped.

Visual variable

Linear

Areal

$21 / 2-D$

True 3 - D

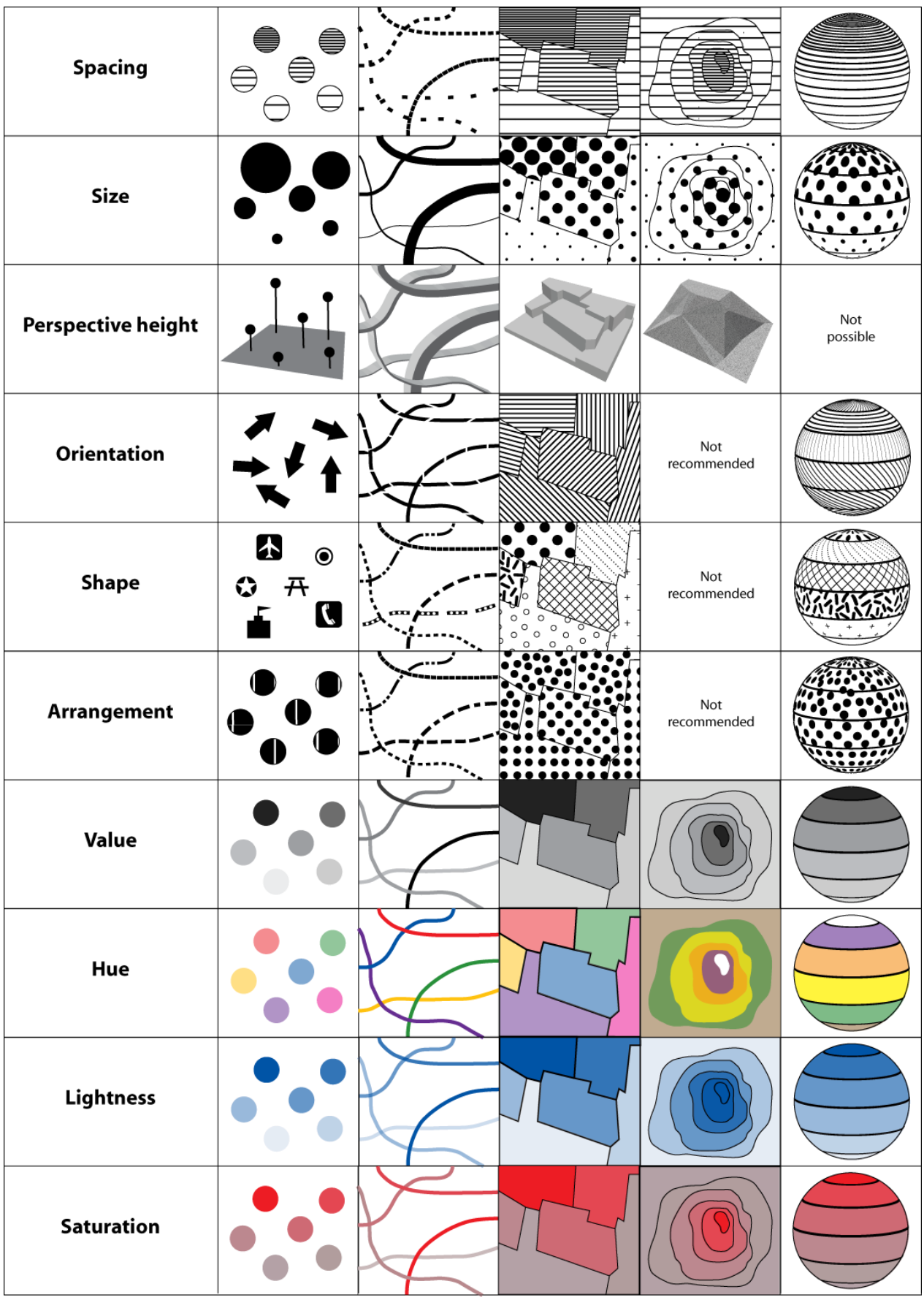

Figure 11. Visual variables 
Visual variables can be sub-divided into those that differentiate and those that order. Differentiating visual variables should be used to show qualitative difference between features while ordering visual variables should be used to show quantitative difference between features.

- Spacing visual variable refers to alterations made in the distance between the various individual marks that make up the symbol. For example decreasing the spacing between the hatch lines of an area pattern, or increasing the dash spacing of a dashed line.

- Size of graphical objects can be used in a number of different ways in cartography. The actual size of the object itself can be altered which both the point and linear examples in Figure 11 illustrate. Alternatively, the size of the marks that are used to create the symbol can be altered. Changing the size of either the whole symbol or the marks that make up a symbol is used to reflect quantifiable differences in the data on ordinal and numerical scales. The visual impression is one of a measurable difference in the magnitude of the underlying feature being mapped.

- Perspective height refers to some sort of perspective view of the data being mapped. With more map products being created in perspective form (tilted map surfaces for instance) then perspective visual variables are becoming increasingly important. For point features, each individual feature is given a raised stick (or lollipop) where the length of the stick itself is relative to a quantifiable aspect of the data on the ordinal or numerical scale. Linear and area features are depicted as some form of extruded box-like structure to give the impression of magnitude. This is most appropriately used to depict quantitative data on a numerical scale. Beware also that as the map is in some sort of perspective orientation, this means that features closer to the front of the map are shown at a different scale than those towards the back. This can create problems of comparing like-for-like across the map.

- Orientation visual variable is defined by the angular arrangement of the marks that make up the symbol. It is inherent in the symbolization for point features as the whole symbol is oriented. Orientation best serves the display of qualitative data on a nominal scale of measurement. Each of the symbols (per feature type) in Figure 11 is equal in visual perception so changes in orientation connotate difference as opposed to quantitative difference.

- Shape works in a very similar way to orientation. Again, it is best suited to point phenomena (through the design of either geometric or mimetic symbols) as opposed to linear, area or true 3-D. It is also used to show nominal difference in data and cannot be effectively used to illustrate quantitative difference. Patterns created for area features are sometimes mimetic and are commonly used e.g. on geological maps to illustrate structural form.

- Arrangement of the marks that make up symbols for area and true 3-D can vary either geometrically or randomly. For linear features, arrangement of dots and dashes can form certain sequences. For point phenomena, the position of a particular graphical mark within the overall point symbol constitutes arrangement. In practical terms, the use of arrangement is very similar to both shape and orientation; it should be used for representing nominal difference but not numerical data.

- Value refers to the extent of darkness and lightness of a particular shade when other dimensions, such as hue, remain constant. In the example in Figure 11, the grey scale has been used to illustrate change in value for all features. Lightness is most effective at representing ordinal data since it connotates some measure of quantitative difference between features. Darker values are seen visually as representing greater quantities than features represented by lighter symbols. Value should not be used as a differentiating visual variable. While it is commonly used for statistical mapping (e.g. choropleth mapping), value is only marginally effective at representing numerical data. The importance of classification and also the visual steps between symbols are important to achieve an effective representation but even then, it is not possible to accurately determine a measured value from the symbols alone.

- Hue refers to the dominant wavelength of light that forms our color association. What we commonly refer to as the colors of yellow, red, blue or green are more accurately referred to as hues. In many ways, hues are distinct symbols and should not be mixed in a scheme that is representing numerical data. Hue is not particularly effective at displaying quantitative difference in its own right although it is often used in combination with other visual variables to emphasize ordinal differences. For instance, a road classification system might employ different line sizes to indicate order but combined with hue as a secondary visual variable to improve the representation. Hue has been used for numerical data representation despite its marginal effectiveness. For instance, atlas mapping conventionally deploys a range of alternative hues to 
depict relief. This is referred to as hypsometric tinting. In qualitative terms, hue is often used to show nominal difference, for instance to provide a representation on a political map that must illustrate the delimitation of different countries without implying that one is more important than another.

- Lightness operates in exactly the same way as value but rather than using the grey scale, a particular hue is held constant (e.g. blue in Figure 11).

- Saturation is also referred to as chroma and is described as a mixture of grey and a pure hue of a dominant wavelength. Saturation is, in fact, the intensity of a hue as lightness is held constant. In Figure 11 a red hue has been used and lightness held constant as intensity goes from a desaturated grayish-red to a fully saturated red. Saturation can be used in a similar way to lightness for showing quantitative differences in data although lightness is often preferred to saturation on the grounds that it is more aesthetically pleasing.

\subsubsection{Using Visual Variables}

The symbols depicted in Figure 11 provide only a simple diagrammatic representation of the general use of visual variables. The range of choice in designing effective symbols using visual variables is almost infinite. Indeed, one of the main ways in which symbols can be varied is to combine two or more visual variables, conventionally using a primary visual variable combined with a secondary visual variable. In this sense, the visual variables illustrated in Figure 11 act as the basic building blocks for more complex symbolizations. Figure 12 illustrates the combination of size and spacing to create an area scheme for displaying quantitative difference.

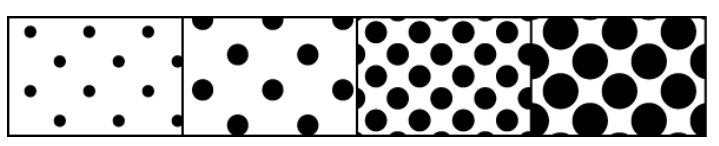

Figure 12. Combining visual variables

Design and deployment of certain visual variables has to be done with respect to the specific mapping task undertaken. One map might be more appropriately designed using lightness to depict quantitative difference yet on another, size might be more appropriate. The mapping context and map type are extremely important considerations which inform choice in visual variable selection.

\subsubsection{Mimetic Symbols}

Mimetic symbols have been mentioned in the discussion on shape. A mimetic symbol (sometimes called a pictograph) is one that is designed to look like the feature it is being used to depict. A mimetic symbol can be simple in design or more pictorial and illustrative. Figure 13 illustrates a range of alternative mimetic symbols that are much simpler and often deployed on topographic mapping or general reference maps. They attempt to capture the character of the feature to be represented by designing a symbol that is graphically linked to the feature itself. They differ from geometric symbols which take the form of standard geometric objects (square, circle, triangle etc.)

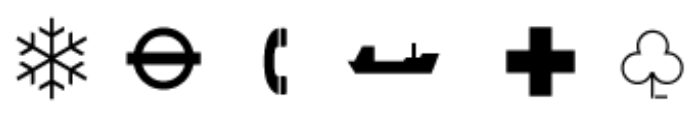

Figure 13. Mimetic symbols

\subsubsection{Bivariate and Multivariate Symbology}

Bivariate symbology is a type of thematic map symbology to be used where a map portrays two variables at the same time for each feature in a class. Multivariate symbology extends this to more than two variables, but is much less used, as it is more difficult to keep the variables distinct but readable. Bivariate mapping can be particularly useful to visually present the correlation (or lack thereof) between the two variables (e.g. vegetation and rainfall; or family income and ethnicity).

Care must be taken to choose the cartographic criteria carefully to ensure that both variables are distinctive. Examples of appropriate ones might include:

- For polygon data - show one variable shown using a color fill, and the other using hatch density or dot density. Or for one use color hue and for the other use color saturation. Or for one use color intensity for Cyan and for the other use color intensity for Magenta, to be combined into a final color. 
- For line data - show one variable using line thickness, and another using color.

- For point data - use two sizes of concentric circle symbols. Or use color as one variable and size as the other.

For multivariate mapping of point data, then a three-variable example might be to use a pie-chart divided circle symbology, with color, radius size, and slice angle. For polygon data, a useful four-variable multivariate technique can be to show two variables as kinds of polygon fill, as for bivariate above, and then show other variables as bivariate point symbols located on the centroid of the polygon.

\subsubsection{Symbolizing Time Series and Animations}

Most GIS data and maps are at a single moment in time (epoch). However it is getting increasingly common for multiple data to be available for different epochs, and the resultant cartographic outputs are required to show the change through time.

The standard ways of portraying time include:

- Multiple adjacent maps, each at a particular epoch - e.g. population density for a city, at the start of each decade for a century, as ten small maps. This allows easy comparison of two or more epochs.

- Similar content, but displayed as a series of superimposed images on a screen. This reinforces the forward flow of time and its effects on the map, but makes comparisons difficult.

Comparing 'now' and 'then' epochs by use of choropleth mapping with a zero-centered range - typically using red density for increase in value and blue density for decrease in value (these are often known as heat maps).

\subsubsection{Legibility}

All mapped, symbolized detail must be legible and clearly distinguishable from other data. If well designed, it should also be capable of being identified without constant reference to the map legend. If symbols are designed in such a way that they are in some way familiar to the map user then familiarity itself assists legibility, information transfer and understanding. Complex symbols decrease legibility and understanding, as does a map display with very dense symbology. A useful online resource to explore the design, arrangement and legibility of symbols is the Map Symbol Brewer by Olaf Schnabel (http://www.carto.net/schnabel/mapsymbolbrewer/)

\subsection{Color}

One of the major challenges for novice map-makers is to employ color in an effective manner. Given that most modern computer systems allow 256 separate grades of color in each of the red, green and blue additive colors that generates over 16 million possible colors. Faced with these possibilities, GIS users need some sort of guidance to make effective choices. Problems arise in matching colors that appear on screen with those that appear on the printed page. Despite automatic conversion between screen and printed colors, they do not necessarily match and maps are often created that are based on the map-maker's perception of color as it appears on screen with the printed map being a poor relation.

\subsubsection{Additive and Subtractive Color Mixing}

There are two major systems for mixing color, depending on whether you are mixing light sources (RGB), or ink pigments (CMY or CMYK). Figure 14 illustrates the RGB and CMY systems.
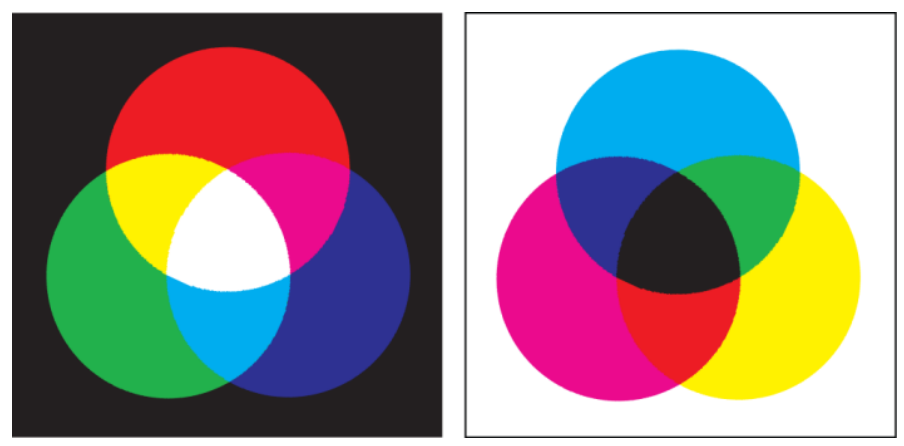

Figure 14. (a) Additive RGB color mixing (b) subtractive CMY color mixing 
The process of mixing colors using the primary (Red, Green, Blue) colors is referred to as additive mixing. If three lights have red, green and blue filters attached they create beams of red, green and blue light. Where the three beams overlap this creates reflected light perceived as white. Where only two beams overlap, the reflected light produces a mix of color. Blue and green together form cyan. Blue and red form magenta, and red and green produce yellow (Figure 14a). By altering the intensity of each of the beams of light, different colors can be created since RGB will be mixed in different ways. Additive color mixing is used in computer screens: minute phosphor dots arranged on a screen emit red, green and blue light in varying intensities. From a distance, the varying intensities reveal a sum of light and, consequentially, a color. The background color on a screen is black which represents no color emission.

Printing on white paper is achieved by using subtractive color mixing and three transparent inks: cyan (C), magenta (M) and yellow (Y). Cyan transmits green and blue, magenta transmits red and blue and yellow transmits red and green (Figure 14b). For example, if yellow ink is printed on top of cyan ink on white paper the yellow ink absorbs blue light but transmits red and green; the cyan layer then absorbs red light so only the green light reaches the paper and is reflected back to the observer. This printing method is the basis of the offset printing process and also color printing from computers using inkjet or laser technology. CMY colors are referred to as the subtractive primaries or process colors.

Because they too are not completely pure (i.e. they do not absorb or transmit $100 \%$ of the theoretical wavelengths in practice) then placing the three colors on top of each other does not produce pure black. Hence a black $(\mathrm{K})$ ink is usually used as a fourth printing color. The $\mathrm{K}$ means key since black is normally printed first and other colors keyed or registered to it.

\subsubsection{Color Specification - Hue, Lightness, Saturation}

No two people will necessarily perceive color in the same way. One person's description of blue may be based on the perception of a slightly different color to the blue that another person recognizes. Notwithstanding problems associated with color blindness, most people with normal color vision tend not to differ substantially in their color perception. In map design terms we can therefore prepare maps based on an assumption that the map user has average color perception (unless, of course, we are designing maps for specific purposes such as for color blind users). This being the case, several systems have been developed to describe color based on average observers.

These systems have to be more detailed than simply assigning a specific color to the label 'blue' since the range of possible blues that might be referred to renders the description meaningless. Furthermore, there are only eleven basic descriptors that can be used to describe color, namely black, white, grey, red, green, yellow, blue, brown, purple, orange and pink. Consequently, color descriptions are more precise and are based on the physical stimulation and perception associated with viewing colors. Being able to define color with precision is important in order to control color from input, through graphic display to final printed output. The principle is simple - in map design we select a color (for example from a color chart) and ensure that we use that color's settings throughout the entire design process regardless of what it might look like on-screen. Because we have specified a particular color we can be confident it will look correct when printed.

Our discussion of visual variables earlier provided the basic vocabulary that we use to describe the components of color. Briefly:

- Hue: the extent to which an area is similar to one, or a proportion of two of the perceived colors red, yellow, green or blue;

- Lightness: the extent to which an area exhibits more or less light relative to white (also called value);

- Saturation: the extent to which an area exhibits more or less of its hue (also called chroma).

The three components of color can be altered to provide a range of ways of applying color to map symbolization. What we normally describe as color is, in fact, more correctly termed hue. Green is a different hue from blue (Figure 15 a) and results from different dominant wavelengths in visible light. The range of colors from white through to black (on a grey scale) reflect equal amounts of light at all wavelengths and are, technically, hue-less (or achromatic). 

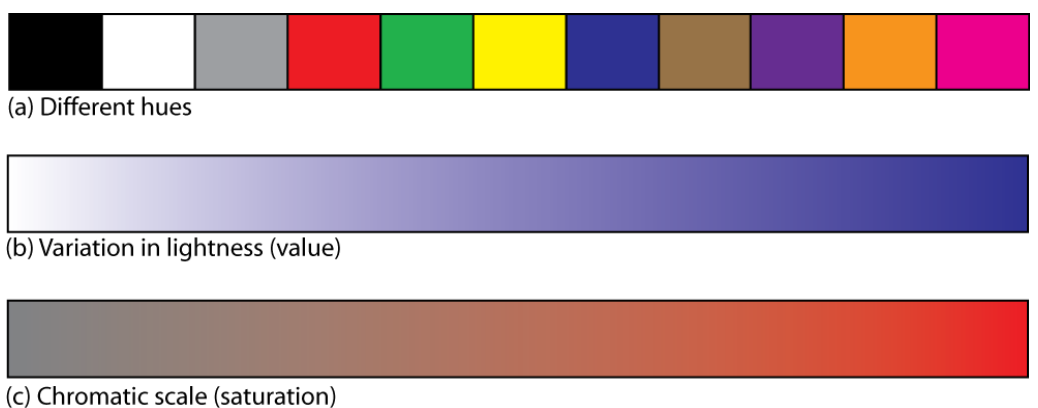

Figure 15. Components of color

For any chosen hue, lightness can vary to provide light and dark versions of that hue (e.g. light blue and dark blue; Figure $15 \mathrm{~b}$ ). It must be remembered though that lightness is a relative concept. If we shine a bright torch at a map then the amount of reflected light increases and the lightness of all hues on the map will also increase in perception although the relative differences between different values of the same hue will remain. This principle does not, however, work if the same light is shone at a computer screen where the increased light is added to the light emitted by the monitor resulting in a decrease in the relative differences in brightness in the screen image. This is one reason why designing color on a computer monitor causes problems and why it is best to work in the same ambient light conditions at all times.

Saturation describes the purity of a hue with the words vivid or dull often used to aid description for high and low saturated hues respectively. A chroma scale, for a fixed hue, is created by taking a hue of high purity and a grey of the same lightness and mixing them in different proportions. The lightness of the scale remains constant across the chromatic scale but the saturation of color increases from grey to full saturation (Figure $15 \mathrm{c}$ ).

\subsubsection{Color Spaces}

A range of color models has been designed for specifying colors that appear on maps. The RGB model specifies color based on the relative intensities of the red, green and blue color of a computer monitor. Each of the three colors range in value from 0 to 255 and can be represented on 3 axes of a cube (Figure 16). The cube itself then becomes a color space such that any point within the cube can be given a value for red, green and blue that specifies the precise color at that point. The CMYK model applies to print mapping where color is created using a subtractive process. Cyan, magenta and yellow are the three subtractive primary colors, with the addition of black $(\mathrm{K})$ being used for true black ink. The three subtractive primary colors are analogous to the RGB color guns and can also be represented as a cube.

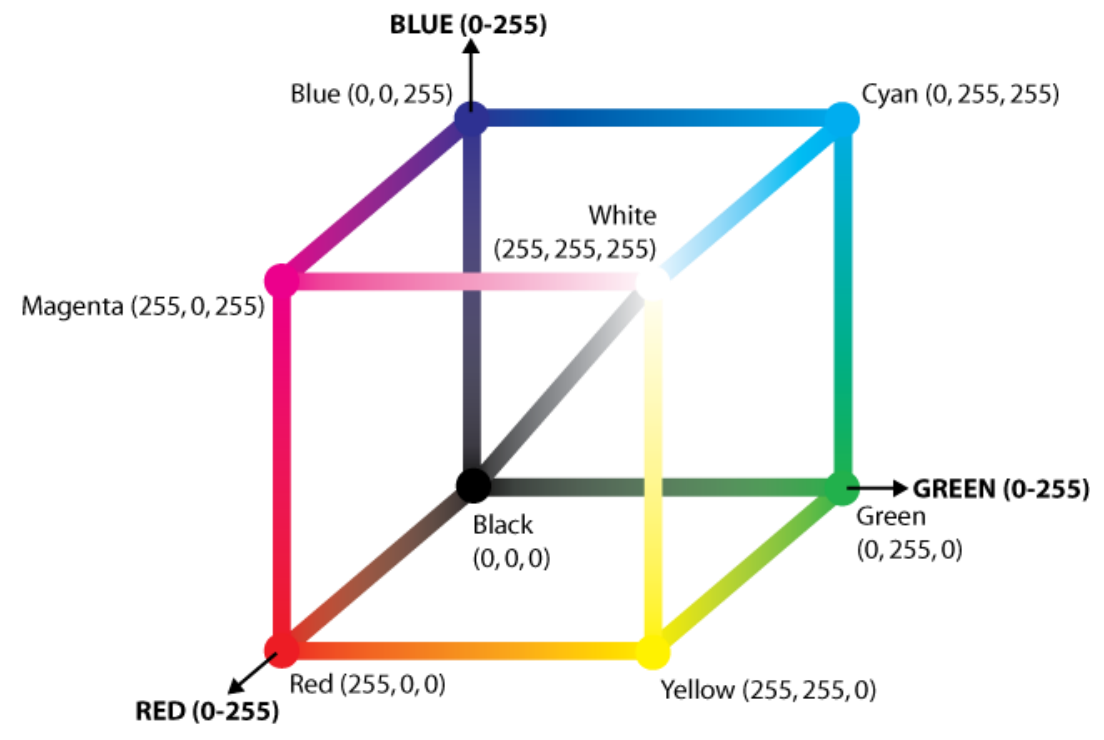

Figure 16. The RGB color cube

The HSV model allows users to work directly with hue, saturation and value. From a map design perspective, this enables cartographers to work directly with visual variables as opposed to proportions of color determined by the technology used for production. 
In a computing environment color is mixed using a model (e.g. RGB or HSV) which is then automatically translated to CMYK for printing. In some GIS you can mix directly in CMYK to specify precise colors for printing regardless of the screen depiction.

\subsubsection{Color Choice on Maps}

Some relationship should exist between the color chosen, the data it is portraying and the meaning to be communicated. Conventions also apply, particularly in topographic mapping such that blue is used for water features, green for forested areas, brown for contours and red for main roads. Note that conventions can conflict, as when the use of green for vegetation can conflict with conventional representations of relief on small scale maps which layer varying greens.

Hue is used as a visual variable to identify map features and to differentiate between features of a different type. To show related features, one would choose hues that are related in some fashion, such as green hues to illustrate different vegetated landscapes. Hue can also be used to provide perceptual distinction. Highly saturated colors provide the greatest perceptual difference. This is one reason why GIS users tend to oversaturate printed versions of their maps - because they are designed on screen and with individual data layers that they wish to perceive differently (Figure 17).

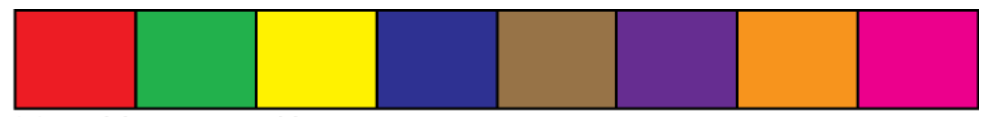

(a) Highly saturated hues

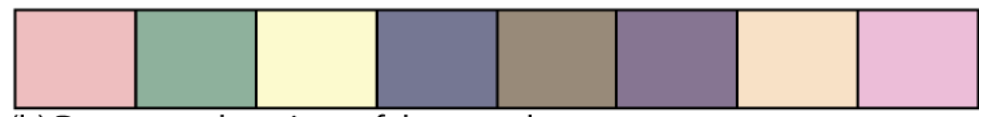

(b) Desaturated versions of the same hues

Figure 17. Saturated and desaturated colors

On a printed map, however, saturated colors are not visually pleasing. A map produced from the colors in Figure 17a a will not be as pleasant as a map produced using colors such as those in Figure 17b. However, this benefit is compromised because inter-color differentiation is more difficult. Consider the difference between the desaturated versions of red and pink, or between blue and purple or yellow and orange. Where a large number of different hues are required, for instance in geological mapping, saturation and lightness should be employed to maximum effect to vary hues in ways that retains distinctiveness.

\subsubsection{Color Use for Quantitative Maps}

Lightness (or value) is used to represent order or numerical difference on a quantitative scale or for large symbol areas. In general, when mapping quantitative difference, larger magnitudes are shaded darker.

The number of visual steps is also important to consider. If a shading scheme has more than 6 hue steps, map users will find it difficult to recognize precisely which class is on the map even when they compare it to the legend. This is particularly true when similar shades are located next to one another.

Quantitative-based shading schemes are generally used on choropleth or isopleth maps to show areas of higher data value compared to lower ones. The problem of adjacent color recognition is more pronounced for choropleth mapping since adjacent enumeration areas may not be adjacent in symbolization terms. On an isopleth map, areas of one class are always nested within areas of an adjacent class so determining values presents less of a problem because colors will always appear on the map in the same sequence as they would in the legend. This gives rise to the principle that choropleth maps should have fewer separate symbols, perhaps four to six separate classes. This is important when a choropleth map includes areas that are extremely small since color perception is strongly modified by size and surrounding color with larger areas of color dominating visual perception. With isopleth maps the number of classes can be much greater because they appear adjacent to one another.

Sequential schemes use lightness to represent data on an ordinal or numerical scale with darker colors representing higher data values and vice versa (Figure 18). 


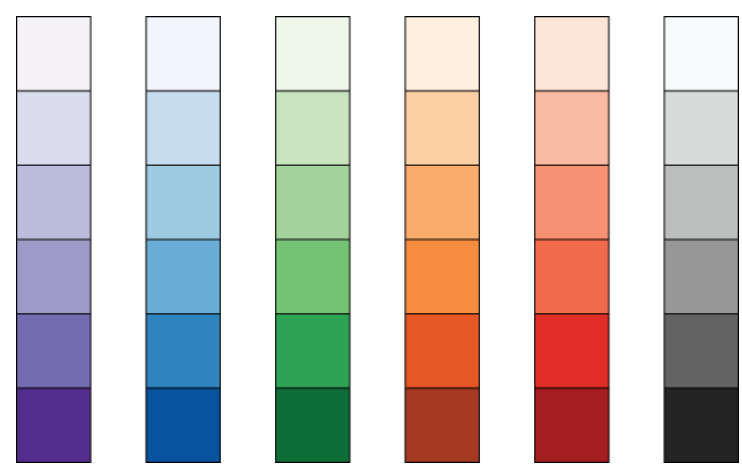

Figure 18. Simple single-hue progressions

Diverging (or bi-polar) schemes are appropriately used where data has different extremes that might be best represented with different hues and a midpoint that might represent some minimal or average value. A diverging scheme emphasizes the midpoint with a light color and then the two extremes with two diverging hues. This sort of scheme can also be used to represent 'no difference' or 'no change' as well. Diverging schemes normally employ a light middle symbol to represent a critical class but this can be discarded to adopt a critical break approach (Figure 19).
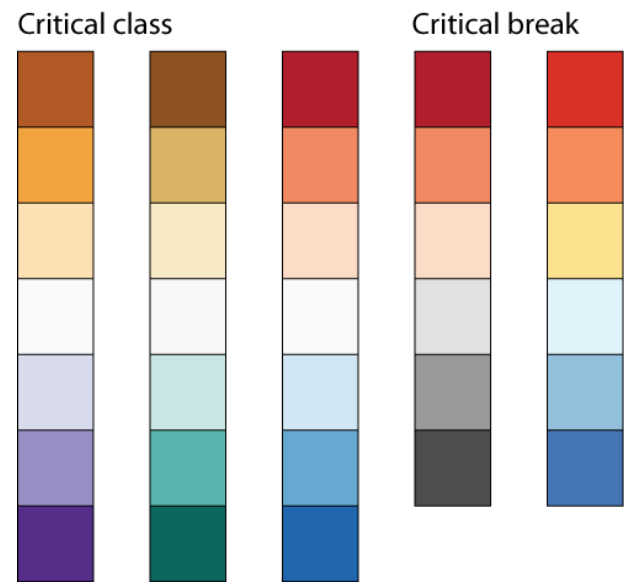

Figure 19. Diverging schemes illustrating critical class and critical break approaches

\subsubsection{Color Use for Qualitative Maps}

Qualitative color schemes should be used to represent nominal data and different kinds of mapped features. These are usually represented using different hues. For instance, a land use map might have a range of different urban, industrial, residential and agricultural land categories and each one needs to be illustrated differently but without giving prominence to any one category or suggesting order in the dataset. The simplest way to achieve this is to ensure the hues maintain a similar contrast with the background color of the map by controlling the value and saturation of each color. This can be problematic on topographic maps where background colors might vary dramatically but it is the principle to be guided by. Logical colors should be used where possible to echo the data being mapped. For instance, different greens might be used to show different types of forested area. Groupings of color on the map and in the legend will help the map reader to gain a general overview by category. Small areas might be symbolized with higher saturation than larger areas to provide an equal perceptual response when viewed.

\subsubsection{General Hints for Color Use}

The following basic design rules also help guide cartographers in creating aesthetically pleasing map layouts:

- In general, avoid very dark or high chroma colors (especially for area symbols)

- Complementary hues form good color matches (e.g. match color for point symbols to complement the background area fill)

- Represent the background of a map with pale tints or grayish colors

- Represent the foreground detail on a map with more saturated or darker colors 
- Complement primary visual variables by the addition of color (e.g. thickness of line weight for road classification augmented by color)

- Avoid emotive use of color (e.g. highly saturated reds for mapping disease)

If color is to be deployed on a map then understanding its construction, perceptual qualities and cognitive associations is vital. There are a number of online resources that help in the selection of appropriate colors for mapping. ColorBrewer 2.0 (http://colorbrewer2.org/) is a very useful interactive web tool developed by Cynthia Brewer and Mark Harrower to assist novice color users to better understand and use color.

\subsection{Text}

The great majority of maps make use of cartographic text to convey information. Typical uses of text on the map are to label places with names (this city is London) or numbers (this Interstate is 10), label areas with values (population of England is 51 million), to describe the terrain ('swamp'), to locate a feature without using symbology ('Well'), or to indicate areas of vague extent ('Bay of Biscay'). Text is also used in titles, legends, and notes panels - more on these in section 11.9 below.

\subsubsection{Typeface, Case, Bold, Italics, Size}

There are several ways to distinguish different kinds of labels on a map. Text can be:

- In a different font or face (e.g. Impact or Lucida Calligraphy). However avoid using too many fonts often a plain sans serif font like Ariel or Gill Sans is clearest. If the map is dense, then using a compressed or condensed font such as Gill Sans Condensed can help fit in more text. Use a serif font such as Times New Roman for blocks of paragraphed text, as the serifs lead the eye through the flow of the text.

- UPPER CASE, Mixed Case, or lower case. UPPER CASE is more prominent (but if used unnecessarily is seen as SHOUTING). Mixed Case (also known as Title Case) is used for most labels on a map.

- Normal, Bold, or italic (sometimes called oblique). Water features such as rivers are often labeled in italic. More important features are shown in bold.

- Plain or underlined. For example those cities that are administrative centers may be underlined.

- Black or colored text - town names in black, water features are often blue, tourist information in red, etc.

- Of differing size -8 point, 11 point, 14 point, or 18 point. Text sizes traditionally are measured in printer's points -72 to the inch.

- Normally spaced or ' 1 e t t e r s p a c e d'. This technique is used to stretch names across polygon areas, such as naming large countries.

\subsubsection{Font Characteristics}

Each font has a set of common elements that assist the description of a typeface (Figure 20).

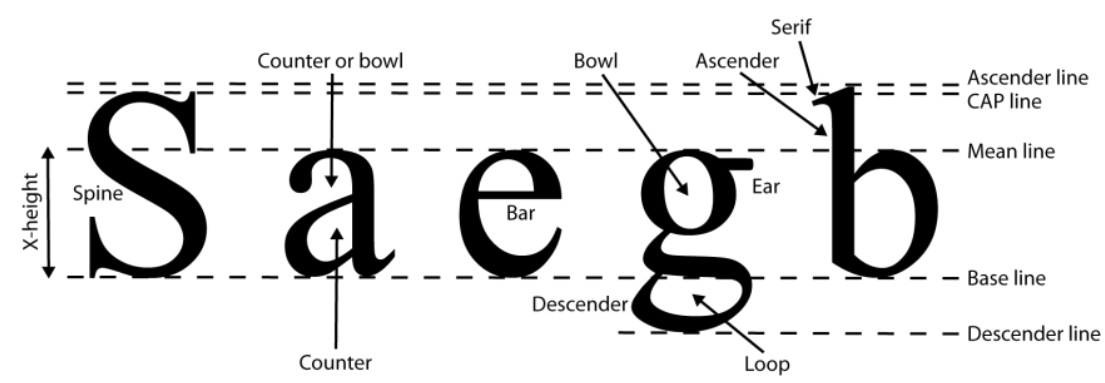

Figure 20. Type elements

A type family refers to a group of type designs that are of common design and share the same basic family name. Figure 21a illustrates a small selection of different type families. For each type family, type can be differentiated by changes to the type style with Roman (normal), Italic, Bold and Bold Italic being common styles with others such as condensed and expanded also being useful in mapping. Figure $21 \mathrm{~b}$ illustrates these 
styles applied to the type family Myriad. Type of a particular family and style is referred to as a typeface (Figure 21c). The word font is used to describe a complete set of characters of type family, type style and size (Figure

21d). A font of type will include alpha-numeric characters, special characters and punctuation marks.

$\begin{array}{llll}\text { (a) Type family } & \text { (b) Type style } & \text { (c) Typeface } & \text { (d) Font } \\ \text { Myriad } & \text { Roman } & \text { Myriad Roman } & \text { Myriad Roman six point } \\ \text { Palatino } & \text { Italic } & \text { Palatino Italic } & \text { Myriad Roman ten point } \\ \text { Tahoma } & \text { Bold } & \text { Tahoma Bold } & \text { Myriad Roman } \\ \text { Bookman } & \text { Bold Italic } & \text { Bookman Bold Italic } & \text { fourteen point } \\ \text { Lucida Sans } & \text { Condensed } & \text { Lucida Sans Condensed } & \\ & \text { Expanded } & \end{array}$

Figure 21. Type characteristics

\subsubsection{Text legibility}

On a map, letters are often spread out across different backgrounds, can be orientated in many ways, might have different leading and are often interrupted by a range of other marks and symbols. The criteria for choosing a typeface are fundamentally that individual letters should be easily identifiable. Studies have found that 3 point type is the smallest that can be discerned by the human eye. However, this is particularly small and assumes 'normal' sight. It is much safer to assume 'average' sight which would give a minimum lower limit for visible text as 5 point.

Lowercase characters are easier to read because they are less blocky in appearance and are often more detailed than their uppercase counterparts which make the overall shapes more visible on a map. The majority of lettering on a map is normally set in Title Case, where each word is composed of an uppercase initial character and the remainder is lowercase. Conjunctions and linking words (e.g. and, on, in, or, with, the etc) are also set in lowercase.

\subsubsection{Label Placement}

Placing text by hand is a slow and painstaking task. Most GIS software has labeling capabilities that will generate text from attribute values and put it next to the feature concerned. There is always a tradeoff between simple but fast labeling algorithms (where in dense areas texts may be on top of one another or of neighboring features) and intelligent label placement engines (e.g. Esri Maplex or MapText LabelEZ) which will work out the best places to position multiple text for minimum conflict. Doing this contextual analysis and conflict avoidance is a computationally intensive task, where intelligent optimization algorithms are needed to avoid the time taken increasing as the square of the number of texts to be placed. Labeling engines allow the user to specify rules for placement, and to weight the classes of features and labels as to their placement importance and urgency of avoidance of overprinting.

\subsubsection{Stored Annotation}

Having generated labels using a dynamic labeling engine, once you are satisfied with the map content, you can convert the dynamic labels to stored annotation. This freezes the placement, and is then much faster to draw as it does not need to do the conflict analysis for every screen redraw. In addition, human intelligence can be applied if necessary to move manually a few texts to improve clarity. However if the underlying features are edited to move them or change attribute values, then the text may not automatically update until regenerated. Hybrid solutions exist like the ArcGIS 'Feature-linked annotation', which will automatically update annotation if the attached feature is changed. It is also worth noting that labeling engines only extend to mapped labels and not titles, legends and other marginalia.

\subsubsection{Placement Preferences}

When placing text manually, or when setting up the rules for automated placement, there are various 'best practices' that have evolved over time. The 'bible' of this is the article [Imhof 1975]. These rules include the following: 
- Label points to the right but slightly above the locating point (avoids confusing a dot symbol with a letter of the label). If this location would cause conflict, then the sequence to try is below right, above left, below left, centered above, centered below (Fig. 22).

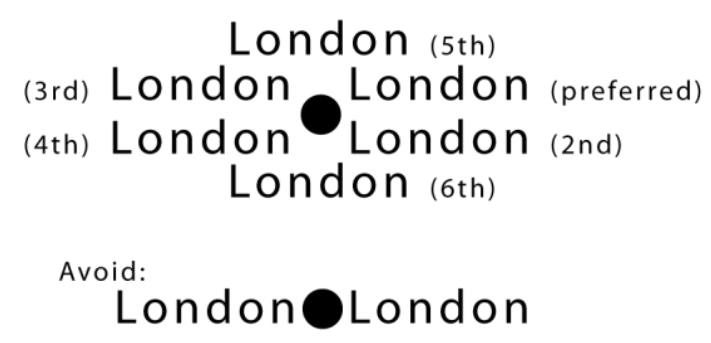

Figure 22. Preferred label placement for point features

- Label lines such as rivers above the line if possible (else below) and parallel to the trend of the line in a section where the text can be read without turning the head too far. Avoid text being upside down, or running right to left (Fig. 23).

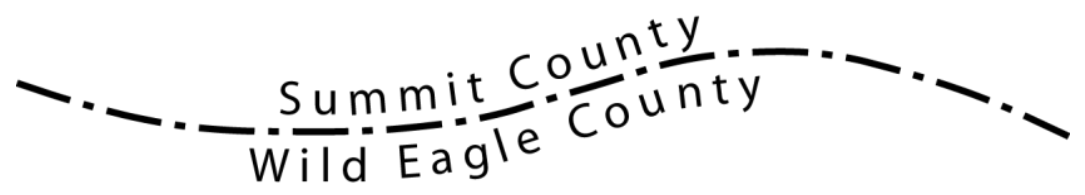

Figure 23. Preferred label placement for linear features

- Label areas horizontally, unless the polygon is shaped to be much longer in another axis. In this case think of curving the text along the long axis of the polygon and possibly letterspacing it so that it stretches across the whole area (Fig. 24).

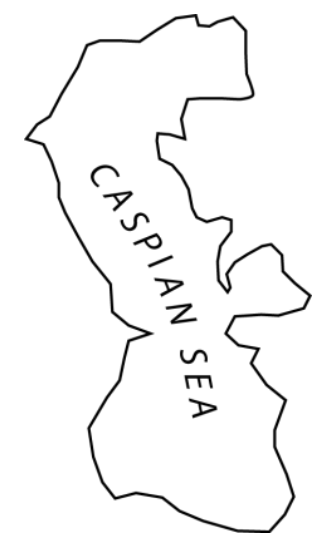

Figure 24. Visually centered area label with appropriate orientation

Where possible, avoid placing type over other graphical marks or map components, particularly when black type is placed over the top of black linework such that the type is difficult to read. Figure 25 illustrates the problem of conflicting type and linework. Where it is not possible to place the type to avoid such overprinting, techniques exist to mitigate the impact. These include filled outlines or halos which are the placement of a block of color or a buffer around the text so that it draws underneath the type but over the linework. A more sophisticated approach available in some GIS is selective masking, which erases only specified underlying components, e.g. erasing a black road casing but not a red road infill.

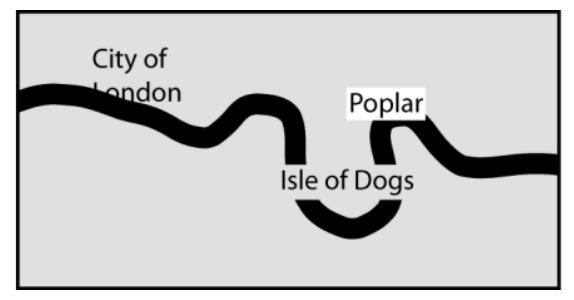

Figure 25. Overprinting and the use of simple masks 
A useful online tool for exploring design and placement of text is Typebrewer (http://www.typebrewer.com/). The tool allows users to experiment with typographic alternatives to see the impact that different approaches have on the overall look of a map.

\subsection{Associating Symbology with Feature Data in GIS}

In addition to the various conceptual design considerations that govern the development of appropriate symbols for a map, consideration also needs to be given to how such specifications are stored. There are a number of different ways that symbology can be associated with the feature data in GIS which bring different advantages in different circumstances.

Traditional GIS architecture is to separate the storage of feature data (geometry and attributes) from symbology (colors, widths, icons), so feature data is stored in a database, but symbology is usually read from lookup tables or style files. This has advantages of being able to reuse and share the geographic data with different symbolization in different projects.

The connection of a feature to qualitative symbology (showing 'what is here?') is usually by matching of one or two attributes. For instance, a road class may have one attribute indicating road classification (interstate, divided highway, highway, major road, minor road, street, track), and another attribute indicating properties such as under construction, or disused. Then distinctive symbolization is applied to the meaningful combinations - e.g. "Major road under construction" as a red dashed line of specified width and dash spacing.

Quantitative symbology (showing 'how much' of a quantity) normally uses the values of a single attribute. If this is a small integer, then the individual values can be assigned symbology directly. (e.g., different size symbols for number of children in a family). In the case of a real number or large integer attribute, this has to be classified into a smaller set of symbol classes. Class breaks can be set manually, or most GIS provide a selectable set of methods for automatically suggesting class breaks, the best known being Jenks Natural Breaks [Jenks 1967]. As an example, if the feature class is cities, and the attribute is population, then they could be symbolized with five sizes of symbols for different population cities (less than 1M, 1-2M, 2-5M, 5-10M, above $10 \mathrm{M})$. It is good practice to choose breaks at round number values.

Quantities may also be normalized against another attribute before classification and symbolization, e.g. the number of children in each district, normalized against total population in the district.

\subsubsection{Production Symbology using Rules and Overrides}

For production cartography of high-quality published maps, although the same association mechanisms can be used, it can be frustrating to have to set up rules for every eventuality. This is particularly true where an association might be relevant for the majority of symbols in a particular feature class, but where there are a few instances where a slightly different graphical treatment is necessary. In production cartography, the ability to be able to define general rules but also cater for special circumstances is crucial to the development of an efficient production workflow that doesn't require constant reinvention.

The ability to override rules for particular features, and hence give graphical freedom to the cartographer has been central to the development of new tools for symbolization. One exemplar of an implementation to support these 'rules and exceptions' is the Cartographic Representations mechanism of recent Esri ArcGIS releases. This has been used to great effect by the Swiss national mapping agency swisstopo to produce its prestigious national map series from GIS data, rather than in a graphics program, while maintaining its exacting standards. To do this, swisstopo stores the symbology (both rules and overrides) in the same database as the features. In this way it can share standard symbology across an enterprise, but be able to apply human freedom to override the machine, to achieve optimum clarity. In this way, the mapping agency can develop general rules for the symbolization of features across map series but also allow individual cartographers to make adjustments, using overrides that are stored in the database. The storage of rules and overrides in a database provides a substantially more flexible and efficient approach to symbolization in a GIS environment.

\subsubsection{Programmatic Symbology}

Some GIS have mechanisms by which portrayal of a feature class is determined not by lookup of symbology, but by invoking a specific user-supplied software procedure. One example of this is the 'bespoke renderer' mechanism of Esri ArcGIS, where the user can invoke the extensive ArcObjects software toolkit to determine what and how to draw. This gives great power, at the cost of requiring programming expertise to set up. 
Another example is the 'display method' mechanism of the 1Spatial (formerly Laser-Scan) Gothic suite, which is based on an active object database. Here, instead of the application having fixed rules about representation, or using a static table of styles, all drawing is done by the application sending a message to each relevant database object saying 'draw yourself'. The response to the message is to execute the object's 'display method', which can use the power of the object database and spatial toolkit to decide what to do. It still has access to a set of representation styles (line patterns, area fills, symbols), but may decide to draw itself differently dependent on scale, surroundings, or any other criterion (e.g. day of the week!).

Hybrid mechanisms also exist, combining lookup of symbolization rules with programmatic extensibility. The 'cartographic representations' mechanisms described in the previous section have the concepts of 'geometric effects'. Geometric effects are small software modules that take the raw feature geometry from the database and modify it before it is used for drawing. Several geometric effects can be chained together, with the output of the first feeding into the input of the second. A set of standard geometric effects are available (offset, shrink, donut, etc) but user-supplied geometric effects are also supported, giving substantial versatility. A similar extensible mechanism exists for controlling the placement of markers as part of symbology (regularly spaced along lines, randomly spaced inside polygons, etc).

\subsubsection{Sequencing Of Drawing Map Symbols}

To achieve a meaningful appearance, it is usually necessary to control the order in which the features in the map are drawn. In more complex cases, it may be necessary to control the individual parts of the symbology (casings and infills of cased roads). In the simplest case, features in the map are drawn in an order which is essentially random within the feature class. The sequence of the classes is usually determined by the order in a 'table of contents' (TOC). The sequence is arranged to ensure that in general, areas are drawn first, then lines, then points, to avoid obliterating detail.

If this standard sequence is not acceptable, then most GIS have a means for assigning numeric priorities to symbol parts for each feature class. The features are then sorted into priority order (features such as cased roads will appear more than once in the list), and drawn in sequence. Advanced capabilities can provide a priority boost to particular features based on an attribute, making it possible to correctly render items like complex road intersections which may have roads going above or below others, as in Figure 26 (data courtesy of swisstopo, cartography by Esri). In Figure 26a all roads are drawn using the same level of priority and the arrangement results in a jumbled set of intertwining features. Clearly, roads are not structured in such a way in reality so by using symbol priority drawing, it is possible to apply a more sophisticated level of control over the way in which the roads are drawn. In Figure 26b the main trunk road depicted in the thick red line casing goes under the vertically aligned black cased road yet there are ramps that clearly join the two roads and which define the junction. On the right of the example, the road order was incorrect so priority was applied to ensure the correct order both to reflect reality but also to provide an effective map representation.

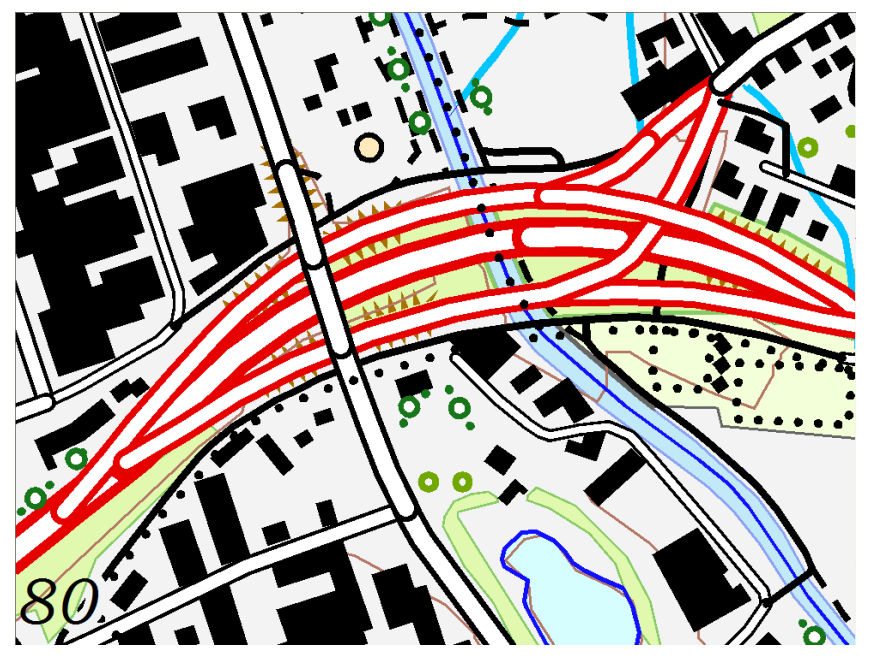

(a) Without priorities

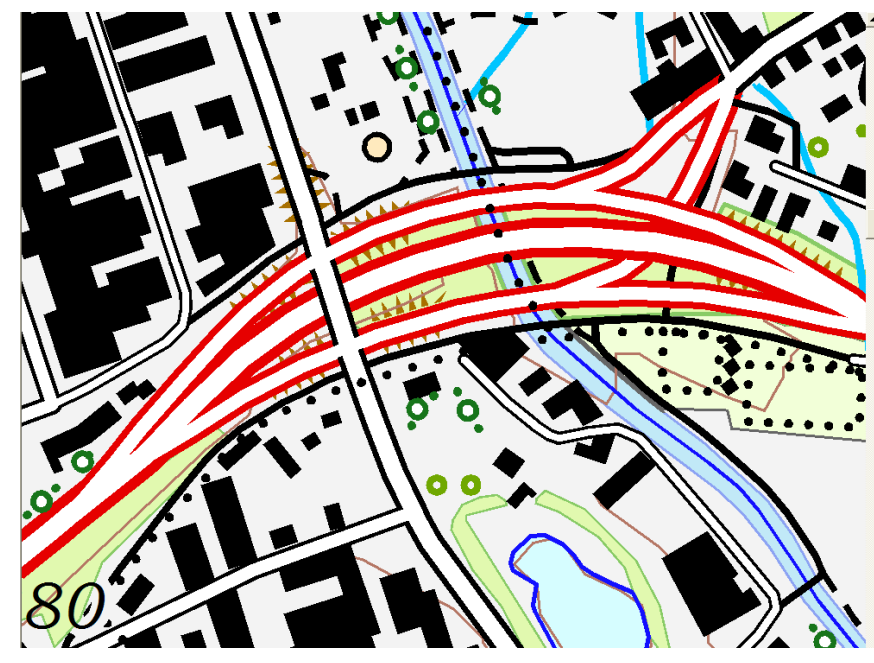

(b) With priorities

Figure 26. Prioritized symbol drawing (data courtesy swisstopo, cartography by Esri) 


\subsection{Relief Portrayal}

The world is not (generally) flat, and relief portrayal is often needed to show how the terrain goes up and down. However, if the terrain is not directly relevant, as is usually the case for very large scale maps or political or thematic mapping, then it is better not to try and show it, as it would get in the way of clearly displaying the important foreground data. The definitive work on relief portrayal is by Imhof [Imhof 82].

\subsubsection{2-D - Contours, Banded Tints, Elevation Models, Hillshading}

The classic simple 2-D portrayal is as contour lines (Fig. 27). If drawn as thin lines in muted (brown) color, this can have the advantage of not occupying too much white space. However many users will have difficulties in interpreting terrain based on contours.

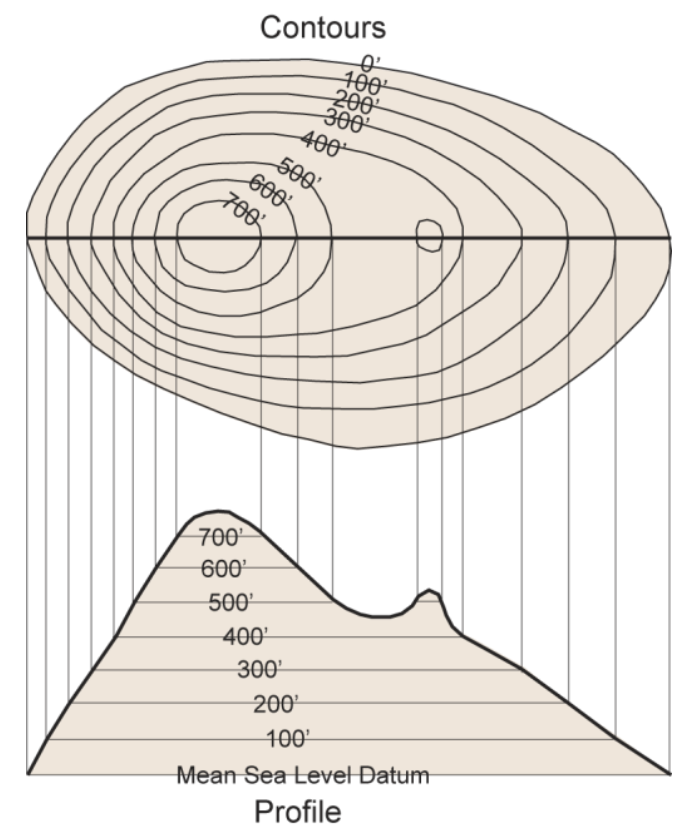

Figure 27. The use of contours to represent height (Courtesy Esri Press)

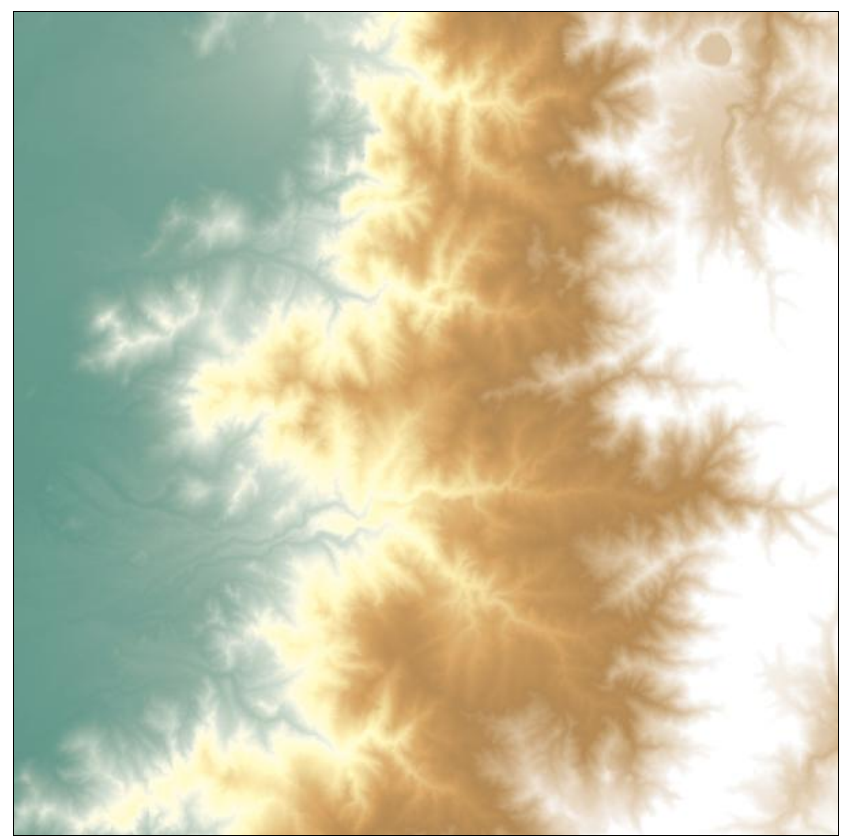

Figure 28. Banded tints to represent elevation (Courtesy Esri Press)

Banded tints (Fig. 28) are related to contours, in that they treat the contours as polygons, and fill them with color. If the colors are chosen appropriately (lowlands in green, going through pastel shades of yellow and brown to grey or white for mountains) then most people can interpret the terrain altitude.

Note that conventions can conflict, as when the use of green for vegetation can conflict with conventional representations of relief on small scale maps which layer varying greens. Hypsometric tinting was developed to mimic green, cultivated lowlands and barren, brown mountains but it does not necessarily suit all landscapes, for example where low lying desert may be fringed by forested mountains.

An alternative approach, when more elevation data is available, is to build a raster digital elevation model (DEM) and apply a similar color ramp to that. Hillshading is an extension to elevation raster, which simulates the shadows that would be cast on the landscape.

Some of the best modern depictions of terrain on maps from GIS cartography use an elevation raster, colored as above, combined with grayscale hillshading - primarily from one direction of illumination, but with a secondary illumination to prevent deep shadows - see Fig. 29. An alternative is to combine the same grayscale hillshades with a land cover raster, to show vegetation etc, as in Fig. 30. 


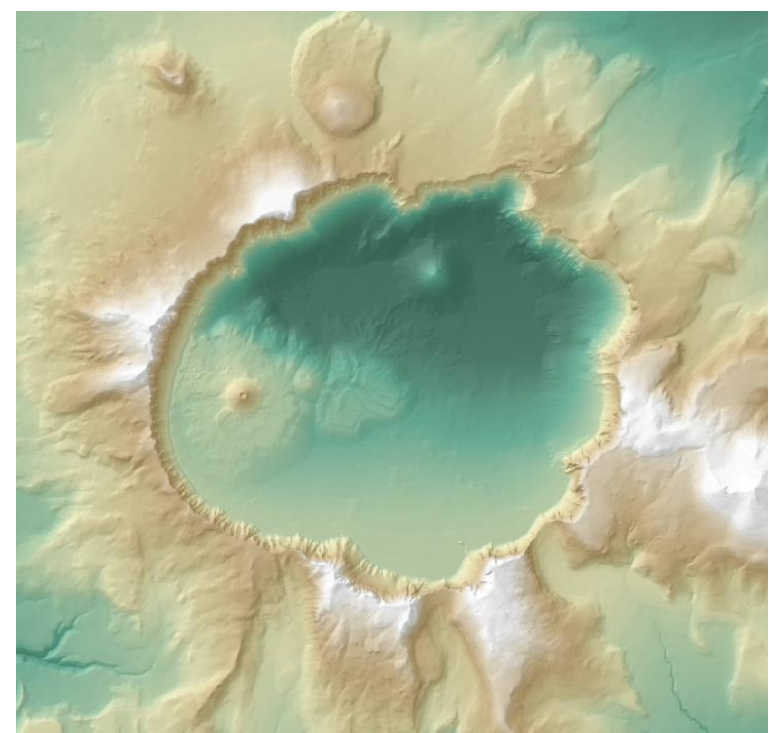

Fig 29 - hillshades plus elevation tint (Esri Press)

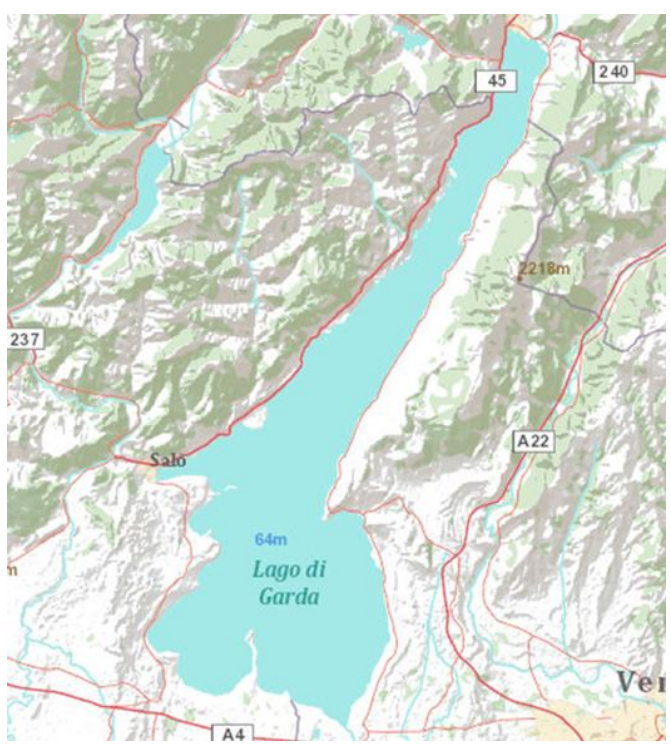

Fig 30 - hillshades plus land cover (Esri)

\subsubsection{5-D - Rendered Terrain Surface and Draping of Cartography}

The next stage of relief portrayal is to switch from a vertical 2-D view looking down on the geography, to an oblique (perspective or isometric) view looking across the virtual landscape (Fig. 31). In this, a digital elevation model is used to create a terrain surface, and the 2-D cartography is draped over it.

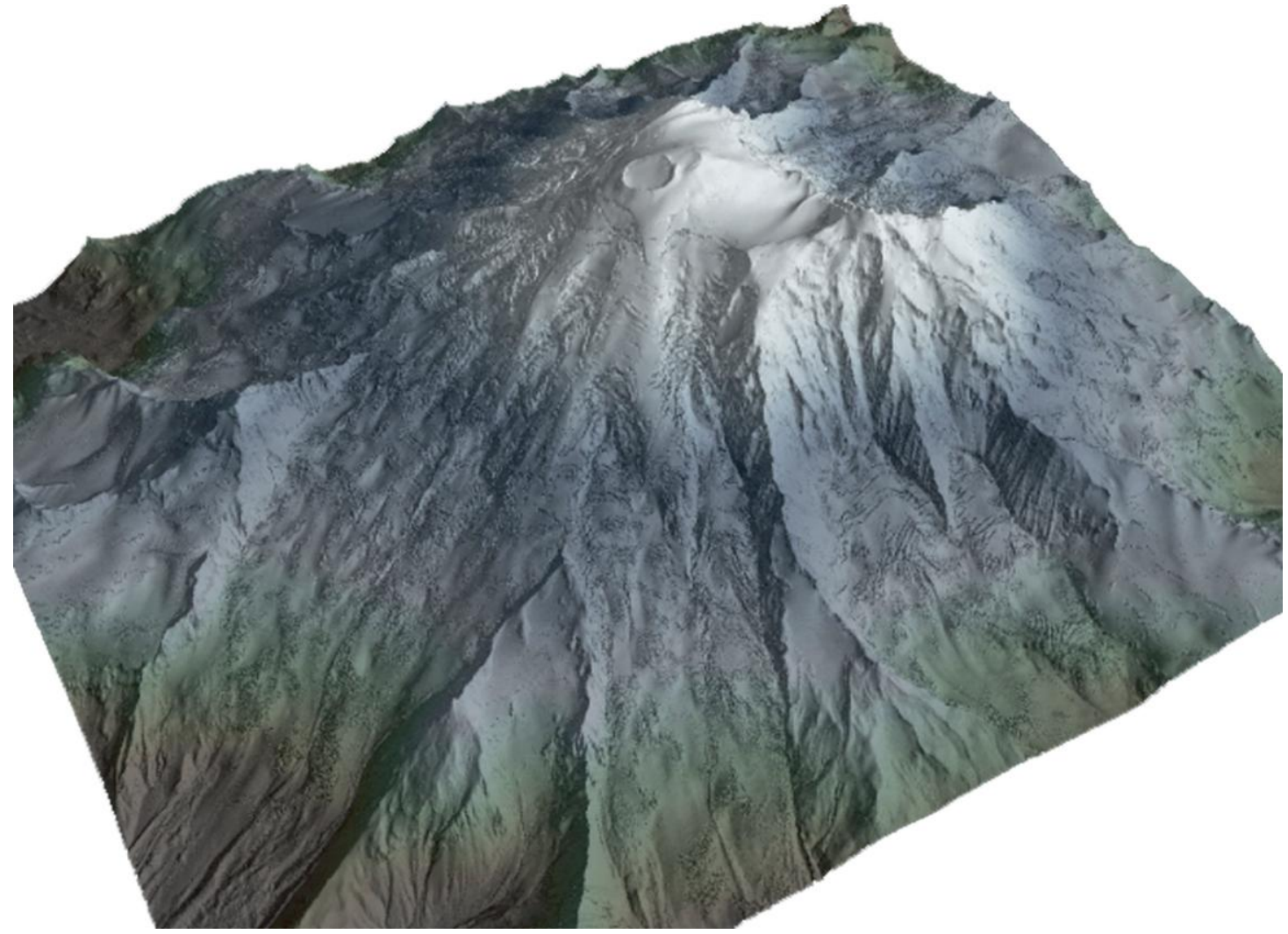

Figure 31. Block terrain of Mt. Rainer, Oregon, from $1 \mathrm{~m}$ DEM derived from ground classified LIDAR returns (Watershed Sciences Inc., courtesy Esri)

\subsubsection{3-D - Immersive Rendering, with 3-D Symbology}

The rise of computer games technology has increased both expectations and the technological resources to do full 3-D immersive rendering of virtual worlds. Here, the digital terrain model is rendered as true 3-D (Fig. 32); using surface textures to make the landscape and superimposed structures look realistic. 3-D symbols can also be introduced, and the user can interact by 'walking' or flying through the model. 


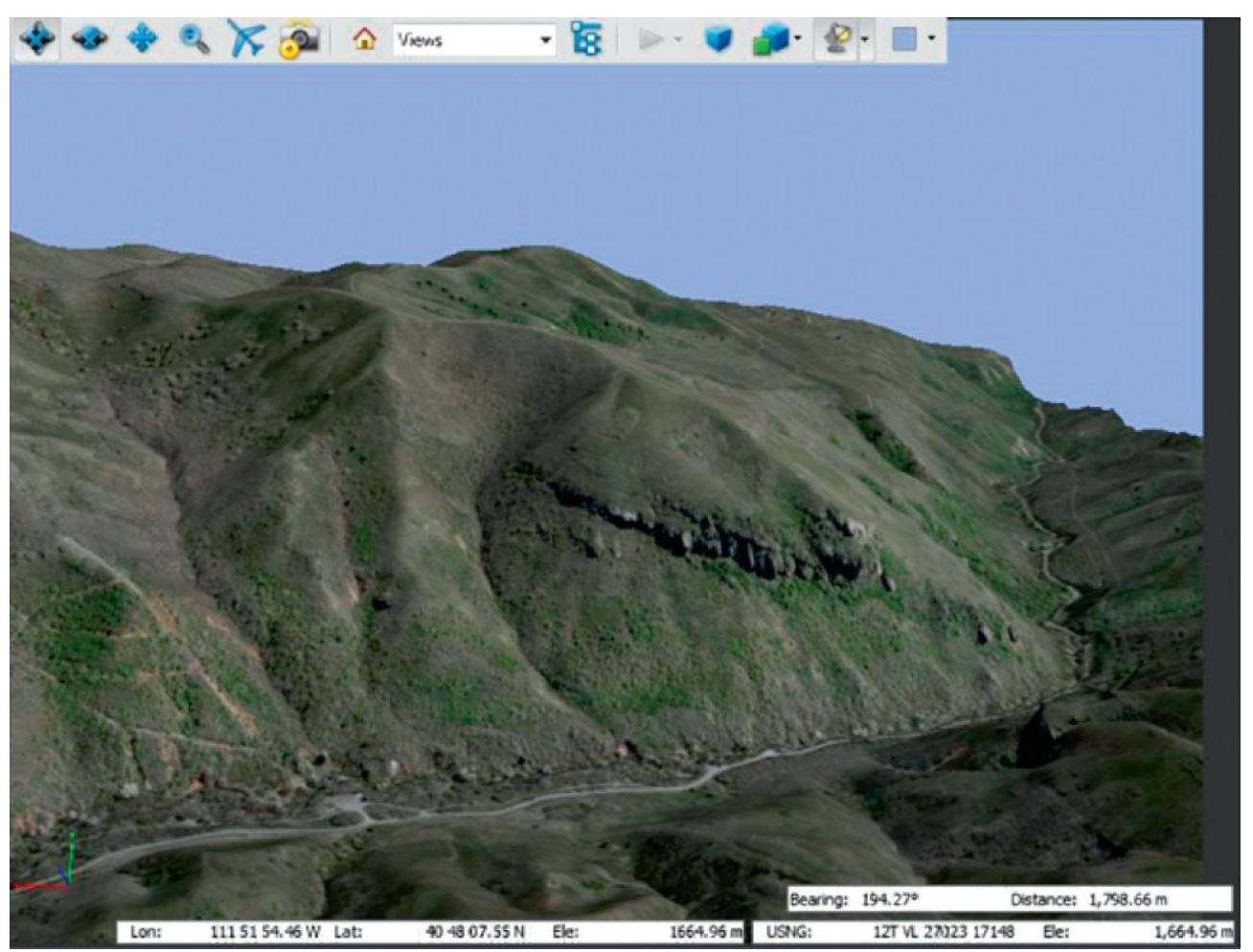

Figure 32. LIDAR derived mountain scene with interactive controls (National Agriculture Imagery Program, LIDAR from the National Elevation Dataset, courtesy Esri)

\subsection{Layout}

Although symbolizing individual features is a major part of cartography, another vital aspect is the composition of a complete communication document. In this, cartography shares many ideas with the remit of graphics design, as done for adverts, posters, brochure pages, magazines, etc. The map image itself as an output of GIS analysis is often only one component of such a graphical product designed to communicate. It is relatively common for GIS users to simply take a screenshot of their data view once analysis is complete, yet this is rarely sufficient or effective. Data views do not provide information on scale and orientation. Nor do they identify useful information such as the projection or data source; they rarely have a title and, possibly, omit a legend altogether. They normally only show the layers of the feature classes as they appear in the Table of Contents (TOC). There is much to be done to take a data view and turn it into a map through a consideration of the way map layers are arranged as well as the paying attention to marginalia and the design of the overall map sheet.

\subsubsection{Marginalia \& Furniture Elements, Insets, Titles, Legends, etc.}

A complete map sheet or page will often have many more elements other than the symbolized and annotated geographic features. Examples include the following:

- Title and subtitle - usually bold and positioned top and central, so that the user is reassured early that they are looking at the right document.

- Legend - explains the map symbology - should only include symbols present in and important to the particular map.

- Scale information - either numeric scale e.g. 1:100,000, or scale bar, or both.

- North arrow - Important if the map is not the usual 'North at the top'; not needed if the map is obviously on a standard grid, nor if it is on a world or continental projection where the direction of North will vary across the map.

- Grid or graticule - a grid is a regular mesh of round-number coordinates - e.g. every $1 \mathrm{~km}$. A graticule is similar but is regular mesh of latitude and longitude lines - e.g. every degree.

- Map border - may be plain or decorative. Often includes the annotation figures for the grid or graticule to help locate the map in the world.

- Logo of creating organization - often mandated. 
- Illustration images - help explain the purpose of the map and make it more attractive.

- Projection, datum description - may not be needed if obviously on a standard national grid, but can be important to avoid misinterpretations - see section 11.3.4 above.

- Credits and copyright note - should include publishing date and author information, plus acknowledgments of contributions of data and advice.

- Commentary - explaining the map purpose, how and why it was produced, expected audience, etc.

- Overview map or location diagram - puts the map into a larger context.

- Inset maps - enlargements of critical areas of the map.

\subsubsection{Visual Hierarchy}

Good map design builds upon the process of deciding the relative importance of map objects including the map but also the marginalia and other map furniture. The graphical representation of this process is referred to as the visual hierarchy. It involves graphically emphasizing those objects which are high in the intellectual hierarchy and de-emphasizing those that are less important. The overall map composition should be hierarchically organized in this way. Additionally, individual map components should be organized according to a visual hierarchy. For instance, in an overall sense, thematic symbols will probably be more important than the title and the legend so emphasis is applied accordingly. Additionally, if all the text is considered as a separate map component then the title is more important than the subtitle and, in decreasing importance, the legend, data source, scale and orientation. Type should therefore be designed accordingly.

Various methods can be employed to create a visual hierarchy. Applying contrast to map components (such as increasing the size or weight of a symbol or emboldening text) can alter the relative attention that the components will attract. The crucial aspect to creating an effective visual hierarchy is to design the map such that the user's eye is drawn to the most important map component first (the figural element or foreground) and the less important ones subsequently (the background). Figure 33a illustrates a map with poor visual hierarchy; the least important components are given relative importance both in terms of the map but also the surrounding detail. Figure 33b, on the other hand, shows a correctly applied visual hierarchy that reflects an appropriate intellectual hierarchy to emphasize the most important map detail but also to de-emphasize marginalia.
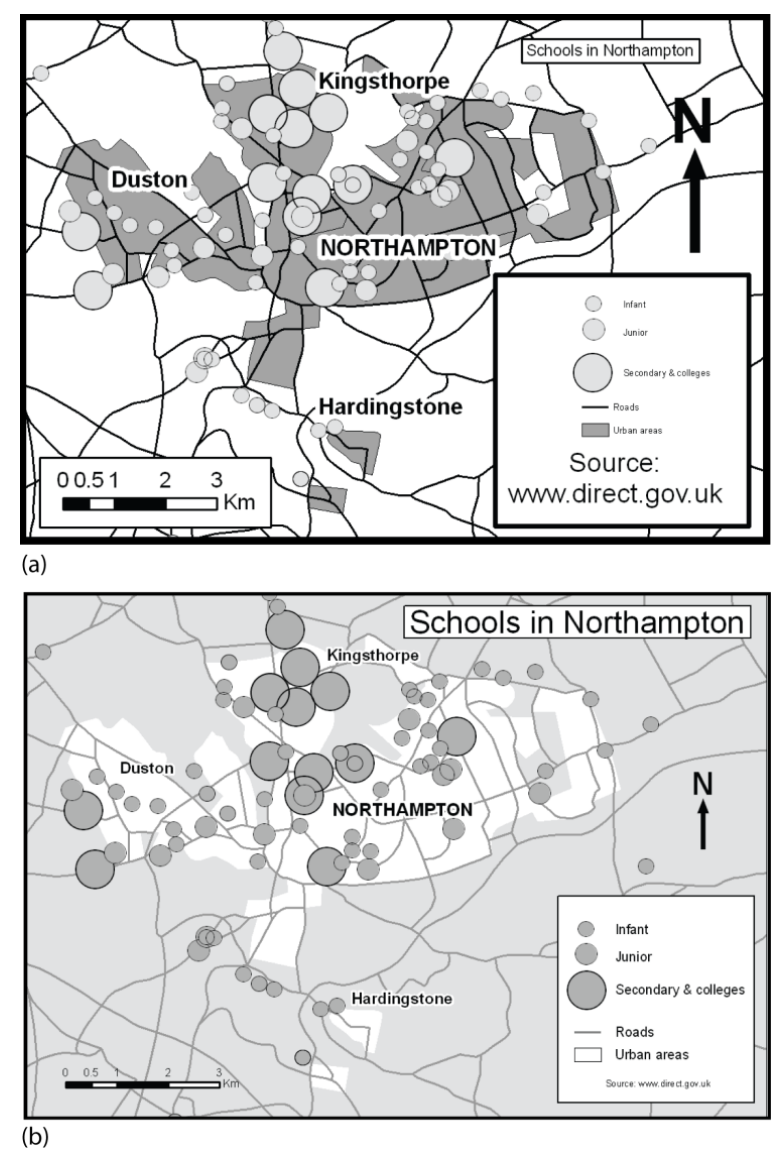

Figure 33. Visual hierarchy (a) poorly applied; (b) appropriately applied 


\subsubsection{Visual Balance and Arrangement}

Visual balance refers to the arrangement of the various map components and empty space on the map page. The objective is to create visually harmony and equilibrium where map components complement each other and work together as a whole. A map which is poorly balanced tends to see individual components competing for space, or have an abundance of poorly used and empty space. The map page should be balanced around the visual centre of the page which is the point just above the geometric centre of the page. This is the point which the eye tends to first and is the fulcrum, or balancing point, around which map components are arranged.

Once a mapped area has been positioned then other map components can be added, usually in order of importance. A well balanced map page does not necessarily exhaust available space, but it does use it efficiently and organizes its map components in available spaces of an appropriate size and position in relation to other graphics. As each map component is added to the page, available space can be re-evaluated. Components will need to be rearranged several times to achieve visual harmony. In so doing, the map page should not be topheavy or 'heavy' in any particular direction. Neither should these areas be cramped or, conversely, too sparse.

In addition to the overall page design considerations there are some more specific aspects to be considered relating to the various map components. These largely relate to positioning, alignment and centering. Alignment and centering of individual map components is normally done through visual estimation rather than precise measurement. When cartographers refer to an object being visually centered they are referring to the process of placing a map component through visual approximation so that it appears centered in the map frame or in a specific area on the map page. Of course, alignment and centering can also be achieved by measurement, particularly when using software to align components precisely, but note that the software may be aligning or centering based on a page dimension which might not equate to visual centering.

Map components are often arranged in relation to imaginary neat lines, with most GIS software having controls for aligning and centering multiple selected graphical components automatically. A useful guide for the design and arrangement of many components on a map page is the golden ratio rule. This stipulates that a ratio of 3:5 (tall:wide) should be a guide for designing graphical components that the human eye finds visually harmonious. For instance, a legend designed in an inset box with a 3:5 ratio will look much more visually pleasing than one in a 1:1 ratio. Similarly, if the legend itself contains inclusions for area symbols, they too should be in a 3:5 ratio. Strictly speaking the golden ratio is an irrational number close to 1.618034 , but $3 / 5$ is a good enough approximation for map composition tasks.

\subsubsection{Page and Map Margins}

Frame lines and neat lines are both types of map or page border that help organize the overall composition of a map and define its extent. A frame line is otherwise referred to as a page border in that it encloses all map components within it (Figure 34a). Consider it as analogous to a picture frame which focuses the map reader's attention on the picture. A frame line is one of the first map components to be placed in relation to the mapped area since it subsequently defines the available space for other map components.

A neat line is sometimes added to a map page inside the frame line to crop a mapped area (Figure 34b). This is not always necessary. Where map detail bleeds off the page the frame line also acts as a neat line, both cropping the mapped are and creating a frame (Figure 34c).

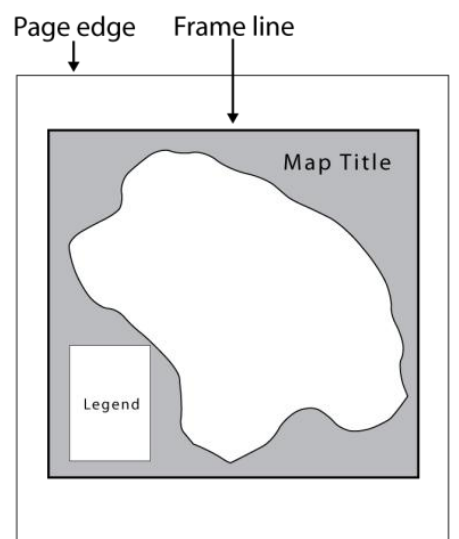

(a) Frame line encloses all map components as a border

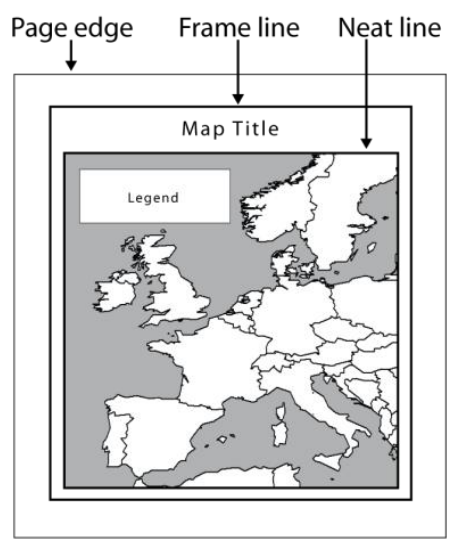

(b) Neat line crops the map area

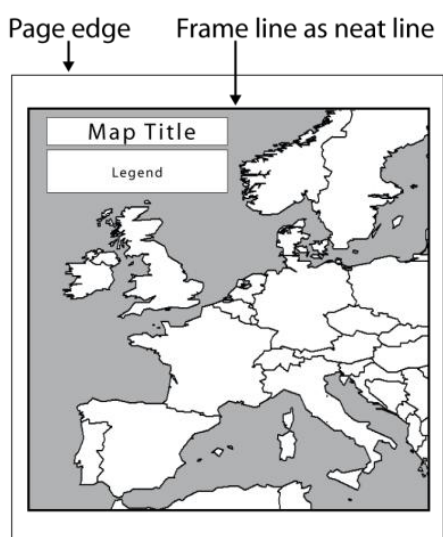

(c) Frame line acting as a neat line

Fig 34 Frame lines and neat lines 


\subsection{Hardcopy and Softcopy Output}

Having got a map looking right on-screen, and having set up an appropriate layout, the next stage is to output it from the GIS, ready for transmission to the consumer. The two main routes to this are as print onto paper, or as a digital image file for download. For printing, there is again a split - low volume printing is usually done directly from the GIS using inkjet or laser printers, whereas high volume printing requires a pre-press stage. The input to this is usually now expected to be an Adobe portable document format (PDF) file.

\subsubsection{Direct Printing}

Direct printing from GIS is common for small-format (A4 or US Letter) or mid-size (A3 or US B) outputs, although there are an increasing variety of large format (A0 or US E) printers available from suppliers such as HP, Epson, or Océ that can be driven from mainstream GIS software. As well as larger format, the higher end printers have multiple ink cartridges with pastel shades in addition to the usual CMYK, to improve color rendering.

\subsection{2. $\quad$ Softcopy - PDF for Download}

Adobe PDF has become the standard format for downloadable finished documents on the Internet. Direct output to PDF from GIS is now ubiquitous, and similar to direct printing. As PDF files are often aimed at on-screen viewing (as well as local small-format printing) the usual color model is RGB. Of particular benefit to GIS users wishing to distribute their work in softcopy is Georegistered PDF; an extension to the Adobe PDF file format from Acrobat 9. The extension adds a coordinate transformation matrix to the file to enable it to be projected and features exist to export layers and other object data directly into the PDF for viewing using a standard PDF browser. This has the attraction that softcopy output can be distributed widely without requiring end users to have GIS installed. It gives end users some capabilities to turn layers on and off, zoom, pan and interrogate object data, which cannot be done with a print product or graphic file.

\subsection{3. $\quad$ Pre-press}

PDF has also become the standard format for pre-press (in the past it was an Adobe PostScript file). PDF files for pre-press are often defined in CMYK colors, or occasionally for some maps (such as navigation charts for reading at night), they may use spot colors (specific named inks). Either way, the printing is then done on offset printing machines, where the paper passes through a set of rollers, one for each ink color.

Separating the composite PDF file into the four CMYK plate images is now usually left up to specialist prepress software; although some GIS have options for doing this rasterisation stage themselves. Handling exact colors, transparency and 'overprint' is a complex area where specialist knowledge is usually needed, but these days many maps can go to print based on a standard PDF output from the GIS.

\subsection{Internet Cartographic Deployment}

Development of maps for internet deployment is a rapidly growing area of cartographic activity. The process of generating a good web map service from available geodata starts with envisioning the product in the light of its purpose, and then authoring a suitable cartographic portrayal, tuned for screen viewing. In the early days of web mapping, this authoring required a programmer to write style files, but these days this stage is usually done visually and interactively using desktop GIS, as it is a very iterative procedure. Similar tools are therefore used to define the web cartography, as to define the appearance of a printed GIS map.

Once the map appearance is acceptable, then the cartographic definitions and links to the geodata are passed by a 'publish' process to the GIS server, which will use them to generate web maps, and to respond to service requests coming in from clients (Internet browsers) through a web server (such as Apache).

\subsection{1. $\quad$ Static or Dynamic Maps}

The next decision is whether the maps are to be static or dynamic. Static maps are generated by the server as a one-off operation, and are then cached as tiles at a range of scales. When a client asks for a map of a particular area, the GIS server retrieves the appropriate tile files and sends them to the client. This is a lightweight, fast and very scalable process. Although the overhead of generating the original tiles is large, its costs are then spread over many users. As well as speed for the clients, it has the advantage that excellent cartography can be 
applied, as the cost of providing a tile with rich (but appropriate) symbology is little different from providing one with very simple symbology.

The alternative approach is dynamic symbolization, where rendering of the geodata into a map is done in realtime in response to each client request. This has advantages for cases where the underlying data is changing frequently. Because much more computation in the GIS server is required for each client request to be satisfied, dynamic maps tend to need to use simplified cartography in order to achieve acceptable response times, and can still be limited in the number of maps per second that can be served to clients, limiting solution scalability.

\subsubsection{Standards}

The Internet is a loosely coupled framework of individual computers, and hence is very dependent on standards. In the area of Internet mapping, many of the standards come from the Open Geospatial Consortium (OGC), which is an international voluntary consensus standards organization, originating in 1994, covering geospatial content and services, GIS data processing and data sharing. The main standards involved in Internet cartography include:

- Web Map Service (WMS) is the protocol for serving georeferenced map images over the Internet

- Styled Layer Descriptor (SLD) is an XML schema for describing how WMS should render a specific layer.

- Symbology Encoding Implementation Specification (SE) is a map-styling language for producing web maps with user-defined styling, used by an SLD.

Beyond the OGC standards, there are also de facto standards arising from the use of common components, whether open source (e.g. OpenLayers and Mapnik, as used by OpenStreetMap and others), or commercial (e.g. Esri ArcGIS with its MXD and MSD map specifications, or Google with KML). Additionally the International Organization for Standardization in ISO 19117 describes a schema for the portrayal of geographic information.

\subsubsection{Web 2.0}

In the early days of web mapping, the clients were very simple, often just a non-interactive map image embedded in a web page. Then came the addition of map controls, to enable zooming, panning and identifying of feature attributes. However the effect of using of such a map control was usually the redrawing of the whole browser page.

In 2004, the "Web 2.0", conference brought together a whole range of maturing technologies, which together made possible the Internet browser as a platform for running interactive applications. Since then, they have been adopted by GIS vendors and service providers, enabling rich mapping applications, without installing GIS or mapping software on the client computer. The main technologies are

- Asynchronous JavaScript and XML (Ajax), a programming style which uses JavaScript in the browser to upload and download new data from the web server without undergoing a full page reload.

- REST (Representational State Transfer) APIs. These are stateless application programming interfaces to Internet services that simplify the building of Internet client applications.

- Adobe Flash and the Adobe Flex framework provided the first rich graphics subsystem within Internet clients, which could handle vector data symbolization and animation.

- Silverlight is a Microsoft framework similar in functionality to Flash/Flex.

These technologies have opened up a web mapping environment within Internet browsers, which is not far behind the richness and flexibility of traditional desktop GIS mapping. Web 2.0 map applications can present a mix of tiled, cached high-quality static mapping, together with foreground vector information, and a user can interact with it through a smooth, dynamic user interface.

In these Web 2.0 mapping applications, exemplified by Google Maps, Bing Maps, Esri ArcGIS Online etc., the map features and layout elements become more responsive and active. For instance within Google maps, if there are several point symbols (pushpins) which are very close or co-located, then if the user moves the cursor over the cluster, the symbols will move apart so that the user can see how many are present, and determine if it is worth zooming in to see them in their true positions. 
Similarly, hovering the cursor over a pin can cause the pop-up of an information panel, describing the facility present at this location. Clicking on a link in the information panel can lead to a video of the location, or a voice narrative. In this sense, much more interaction can be incorporated into the map so that it contains various controls, roll-overs, hot spots and other dynamic tools.

\subsubsection{Designing a Map for Screen}

In one sense there is little difference in the way symbols are designed for display in the computer environment as compared with the traditional paper map, in order to maintain legibility. The same principles of designing symbols to impart useful information remains and the design principles are much the same. However, designing maps for the web can be a challenge as there are both opportunities and limitations presented by the web and the delivery of a map to screen rather than print. The main concerns with the computer mapping environment are screen resolution, map scale, and color.

With regard to screen resolution, the smallest size for displaying a symbol is determined by the size of a physical pixel on the screen. For example, a line symbol representing a road with a line weight (thickness) of 0.15 millimeters, as typically used in conventional cartography, is not a suitable size specification for computer display. This small line weight cannot be displayed on a computer monitor at its true size. The minimum size for displaying a line, point, or area on a computer monitor is the height and width of one pixel. On a typical PC screen there are approximately 96 pixels per inch (ppi). As fractions of pixels cannot be displayed, symbol size specification must be based on multiples of whole pixels.

Map scale is constrained by the size limitations of screens compared with the traditional large extent of sheet maps. This is more extreme on handheld devices, where screen size is minimal, though clearly the publication of a map on screen means that scrolling, panning and zooming are possible to overcome some of the technical limitations caused by the size of the device itself.

Color has been a drawback in web mapping for a number of years due to the constraints of screen technology. Until recently, maps designed for screen were required to be designed according to a web safe color palette - a small subset of colors that can be displayed on most screens. As computers and screen technology have advanced, this limitation has become less important, and the full richness of a 16 million color palette can often be harnessed. That's not to say there are not still problems with color - which out of 16 million colors does one select? The guidelines relating to color choice for maps in general should still be followed to provide good color balance and visual contrast.

Mapping for screen cartography does have advantages though. Maps can make use of a new set of visual variables such as animation, movement (in symbols), shadows, transparency and actions (such as mouseovers or clickable buttons). Furthermore, screen elements can be moveable or dockable. The principal design issue should be to combine a high level of visual interaction with functionality in a design that suits the medium. With new visual variables comes a requirement to make prominent the purpose of the map. This usually requires a decluttering of the map background as well as highlighting the figure to establish an appropriate visual hierarchy.

The use of web objects (widgets) can also enhance web mapping. Such widgets might relate to pull-down menus, hotspots, or moveable map elements. These objects do more than allowing interaction - they also stimulate greater use of a screen map beyond what can be a relatively short attention span associated with web products.

In the same way as cartographers have little impact on how a paper map is to be read in terms of user knowledge and conditions of use, so the same is true for web mapping. It is impossible to know about screen types, system configurations or user actions. Download file sizes might also need to be a consideration to limit costs or time associated with downloading. Such issues can be incorporated into a design by creating smaller, graphically simpler maps. Scaling and zooming options can alleviate some of the problems of designing in a small format, although excessive zooming removes much of the context important to good map communication.

\subsubsection{Map Mashups}

Map mashups have become one of the major growth areas in cartography due to a proliferation of new online map services. This has been supplemented by the satellite-navigation revolution and a reinvigorated interest in maps by the public. The term mashup is used to describe the process of taking information from two or more separate digital, online sources and serving them up in a new combined way in map form. 
In some respects the word mashup is simply a buzzword for the process of map compilation that cartographers have always been involved in. Map compilation requires the sourcing of map data along with the design and production into a useable output format. Mashups are much the same, except they can be produced rapidly by cartographers as well as non-experts using a range of Web 2.0 tools and services.

Google is largely responsible for the rise in the mashup culture and for promoting a democratization of mapping. Google Maps was released in 2005 and at that time comprised a world map consisting of only North America and the United Kingdom - the rest of the world was blank. It was soon 'filled-in' and Google have subsequently added numerous datasets, together with capabilities for individuals to add their own information. The Google cartographic style also broke new ground, being the first such map style to use muted colors and sizes on the assumption that other foreground data will often be superimposed in primary colors.

A drawback for the design of mashups is the need to use standard base maps. Rarely is the published base map optimal for the purpose of the overlayed information, yet this can normally not be modified as it is served from a particular provider. This can sometimes create quite significant visual contrast issues since the base maps have been designed for a particular purpose and are being reused for another. Foreground overlays sometimes need to be particularly stylized or emboldened to ensure sufficient visual contrast. In this sense, many mashups suffer from poor cartography though some base map data (e.g. OpenStreetMap) allows alternative designs to be applied that at least go some way to providing a method of customization.

Map mashups bring the potential for map-making to ever more people. Whereas cartography was once performed by professionals and was subsequently subsumed into the practice of a GIS expert, the rise of mashups means that anyone with an internet connection is capable of combining data from different sources using online maps and services. Consequently, the quality of most mashups up to now has been poor, reflecting the same problems of maps created in the early days of GIS. It is important that those who create mashups pay more regard to the principles of cartographic design to ensure their particular work is represented as effectively as possible. With improvements to online maps and services, some elements of the design process will be automated to help the end user but it remains a challenge for cartography to inform mashup creators of the importance of map design.

\subsubsection{Mobile Mapping}

One of the main drivers of the development of online cartography has been in the mobile market. As our use of mobile communication and computing devices grows, so our appetite for location information is also growing. The paper map used for locating oneself, and for working out how to get somewhere, has often been replaced by ubiquitous mapping on mobile devices, satellite navigation and location aware technology.

Smartphones and Personal Digital Assistants (PDAs) now come pre-loaded with maps and can provide live turn-by-turn information, using live location information to update user position. For instance, Apple's iPhone (and many other smartphones) use GPS position alongside Wi-Fi and cellular tower information to provide a location. This information can be used in conjunction with applications such as Google Maps to derive walking or driving directions based on a cartographic visualization or a synthesized reality view.

The ability for smartphones to provide location information is the driver for a whole new industry, capitalizing on the ability of a phone to be locatable and to report that location. Social networking (such as Facebook and Twitter) is leveraging such capabilities to provide georeferenced information alongside posts. Geotagging of photographs and video is now commonplace so that uploaded media can be displayed and organized as a mashup along with map services such as Yahoo maps.

Built in digital compasses can be used to turn the on-screen map to the correct orientation for navigation and can also be used to tag an image captured using the onboard camera with direction of view as well as $\mathrm{x}$, y position. This can subsequently be used live in 'augmented reality' applications as a base for overlaying other detail such as the location of the nearest metro station and the position of metro lines such as in the Augmented Reality Tube map of the London Underground (Fig. 35). By tilting the phone upwards the nearest stations are displayed along with their direction relative to your location, and distance away. 


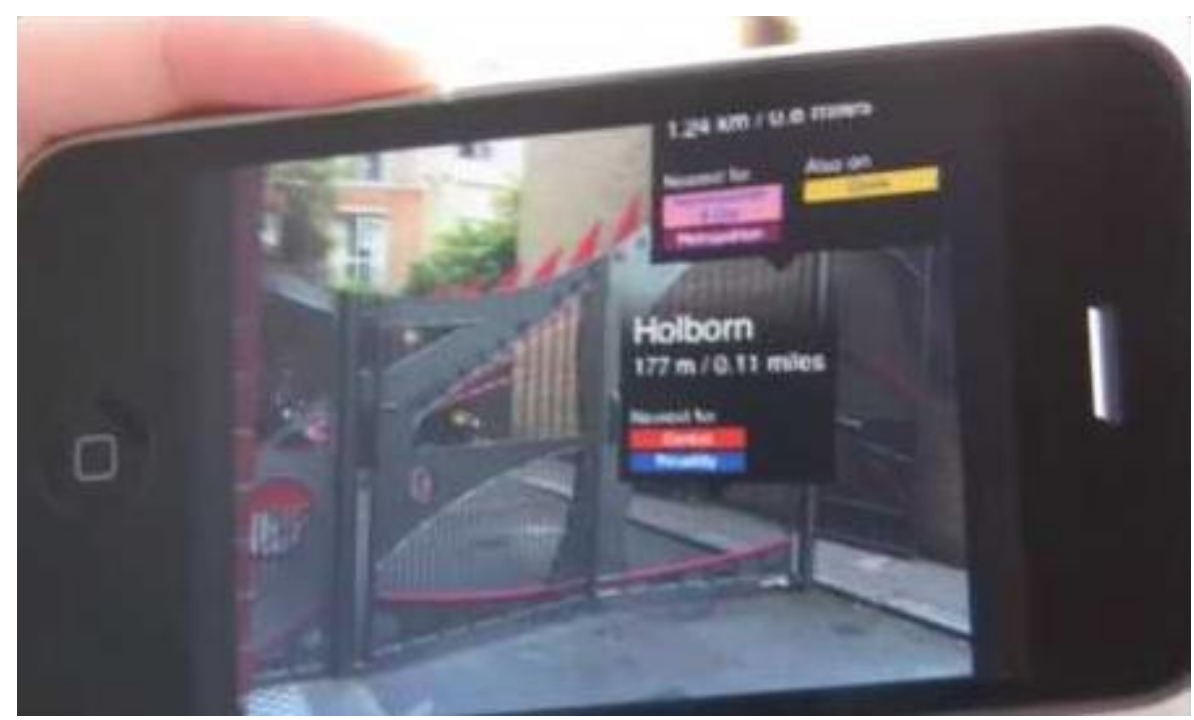

Figure 35. Augmented Reality tube map for London

The design of such augmented reality applications that make use of base map data, mashed-up content and live camera images is at the vanguard of a new mapping revolution that presents challenges and opportunities for cartography. Rather than being an irrelevancy in this age of mashups, cartography has a vital role to play in ensuring that effective design translates into useful and useable mobile applications. The challenges of mobile mapping lie in the small form-factor of devices and the need to be even more proficient in symbol design. Issues relating to bandwidth limitations have been a problem for large-scale uptake, but are diminishing as network technology improves. With ever more competition, the market is opening up to the realization that location is a fundamental part of mobile devices, and appropriate cartography is key to usability and hence to market growth.

\subsection{Further Reading}

- A.L. Allan: Maths for Map Makers (Whittles, Dunbeath 2004)

- C.A. Brewer: Designing Better Maps: A Guide for GIS Users (Esri Press, Redlands 2005)

- C.A. Brewer: Designed Maps: A Sourcebook for GIS Users (Esri Press, Redlands 2008)

- A. Brown, W. Feringa: Colour Basics for GIS Users (Longman, Harlow 2002)

- C. Cauvin, F. Escobar, A. Serradj: Thematic Cartography (Wiley, New York 2010)

- B. Dent: Cartography: Thematic Map Design, $6^{\text {th }}$ edn. (WCB/McGraw-Hill, Boston 2008)

- M. Harrower, A. Robinson, R. Roth, B. Sheesley: Cartography 2.0 (AXIS Maps, Madison 2010), available online at http://cartography2.org/

- M.-J. Kraak, F. Ormeling: Cartography: Visualization of Geospatial Data, 2nd edn. (Pearson, Harlow 2003)

- J. Krygier, D. Wood: Making Maps: A Visual Guide to Map Design for GIS (The Guilford Press, New York 2005)

- M. Monmonier: How to Lie with Maps, 2nd edn. (Univ. Chicago Press, Chicago 1996)

- T.A. Slocum, R.B. McMaster, F.C. Kessler, H.H. Howard: Thematic Cartography and Geovizualisation, 3rd edn. (Prentice Hall, Upper Saddle River 2008)

- J.P. Snyder: Map Projections: A Working Manual (United States Geological Survey, Washington 1987)

- M. Spence, G. Darkes: Cartography: An Introduction (British Cartographic Society, London 2009)

- E.R. Tufte: The Visual Display of Quantitative Information (Graphics Press, Cheshire 1983)

- E.R. Tufte: Envisioning Information (Graphics Press, Cheshire 1990) 


\subsection{References}

- J.P. Snyder: Flattening the Earth: Two Thousand Years of Map Projections (Univ. Chicago Press, Chicago 1997)

- J. Bertin: Semiologie graphique, Paris (1967), republished in translation as Semiology of Graphics (Esri Press, Redlands 2011)

- O. Schnabel: Map Symbol Brewer, http://www.carto.net/schnabel/mapsymbolbrewer/ (accessed August 11, 2011)

- C. Brewer, M. Harrower: ColorBrewer 2.0, http://colorbrewer2.org/ (accessed August 11, 2011)

- E. Imhof: Positioning names on maps, Am. Cartogr. (2), 128-144 (1975)

- B. Sheesley: Typebrewer, A Map Design Help Tool for Selecting Typography (2006) available at http://www.typebrewer.com/

- G.F. Jenks: The data model concept in statistical mapping, Int. Yearb. Cartogr. 7, 186-190 (1967)

- J.O. Muehrcke, A.J. Kimerling, A. Buckley, P.C. Muehrcke: Map Use: Reading, Analysis, $6^{\text {th }}$ edn. (Esri Press, Redlands 2009)

- E. Imhof: Cartographic Relief Presentation (De Gruyter, Berlin 2007)

\subsection{Authors}

Paul Hardy has been providing software tools for mapmakers for over 30 years, and was Product Manager for Cartography at Esri. Kenneth Field was a Principal Lecturer in GIS at Kingston University London, with particular research interests in cartography and visualization, and is now at Esri. He is editor of The Cartographic Journal.

[Paul Hardy, Ken Field, Esri, 2010-2012. Revised 2012-09-07, v11] 